\title{
Physical structure and water line spectrum predictions of the intermediate mass protostar OMC2-FIR4
}

\author{
N. Crimier, C. Ceccarelli, B. Lefloch, and A. Faure \\ Laboratoire d'Astrophysique de l'Observatoire de Grenoble, BP 53, 38041 Grenoble Cedex 9, France \\ e-mail: [ncrimier; ceccarel; lefloch; afaure]@obs.ujf-grenoble.fr
}

Received 12 January 2009 / Accepted 5 August 2009

\section{ABSTRACT}

\begin{abstract}
Aims. Intermediate mass (IM) stars are an important component of our Galaxy, as they significantly contribute to the interstellar FUV field and, consequently, play an important role in the energy balance of the ISM. Despite their importance, very little is known about their formation process and only a few studies have been devoted to characterizing the first phases in the evolution of IM protostars. Here we consider in great detail the case of the brightest and closest known young IM protostar: FIR4 in the OMC 2 component of the Orion molecular cloud complex.

Methods. We analyzed the available continuum emission (maps and SED) through one-dimensional dust radiative transfer calculations. We ran large grids of models to find the envelope model that reproduces the data best. The derived dust density and temperature profiles were then used to compute the gas temperature profile, equating gas cooling and heating terms across the envelope. Finally, we computed the water line spectrum for various possible values of water abundance.

Results. The luminosity of FIR4 has been reevaluated to $1000 L_{\odot}$, making FIR4 definitively an IM protostar. The envelope surrounding FIR4 has a relatively shallow density power-law index, $\sim 0.6$. The most surprising result is that the gas and dust are thermally decoupled in the interior of the envelope, where the dust ices sublimate at $100 \mathrm{~K}$. This has important consequences in the interpretation of the line data. We provide the predictions for the water spectrum, and discuss in detail the lines that will be observed by the Herschel Space Observatory .
\end{abstract}

Key words. ISM: abundances - ISM: molecules - stars: formation

\section{Introduction}

Intermediate mass (IM) stars, namely stars whose mass is in the 2 to $8 M_{\odot}$ range, are crucial in studies of star formation because they constitute the link between low- and high-mass stars (Di Francesco et al. 1997; Mannings \& Sargent 1997, 2000), so can help for understanding if and how different the processes at work are in the two ends. On the one hand, lowmass stars can be formed isolated or in loose groups of a few objects per cubic parsec (Gomez et al. 1993), while high-mass stars are usually found to form in tight clusters (e.g. Hillenbrand \& Hartmann 1998). On the other hand, IM stars are also found in clusters (e.g. Testi \& Sargent 1998; Neri et al. 2007; Fuente et al. 2007), with a smooth transition towards the low-mass star, loose cluster regime for star masses around 3.5 $M_{\odot}$ (Testi et al. 1999). Testi et al. (1999) also concluded that IM stars mark the transition from low-density aggregates of $\lesssim 10$ stars per cubic parsec of T Tauri stars to dense clusters of $\gtrsim 10^{3}$ stars per cubic parsec associated with early-type stars. In agreement with the different observed environments, several authors have proposed that high-mass stars are formed by the coalescence of lower mass stars, whereas other authors favor the "monolithic" formation (see for example the recent review by Beuther et al. 2007). In this context, the IM stars study can greatly help the debate. Indeed, due to their intermediate position, the study of IM protostars will provide crucial information on the transition between the two formation regimes, as well as on the limits of the low-mass and high-mass formation scenarios. Finally, IM stars are among the dominant sources of the interstellar FUV field (e.g. Habing 1968; Gondhalekar \& Wilson 1975), which regulates the phases of the ISM in the Galaxy and in turn, the overall Galaxy star formation process and history. Despite the farreaching importance of IM stars, very little is known about the formation and first evolutionary stages of these stars. The situation is so bad that to date we neither have a satisfying sample of Class 0 IM objects, namely objects representing the first phases of stellar formation where the protostar is embedded in its envelope and its luminosity is dominated by the accretion luminosity, nor a systematic study of their physical structure, as is the case for low-mass Class 0 sources (e.g. Ceccarelli et al. 2007; Di Francesco et al. 2007). This article is the first in a series that aims to fill this gap in our knowledge.

In this context, the Orion Molecular Cloud 2 (OMC2), the closest known region where high- to low-mass star formation is going on, represents a precious laboratory for these studies. Observed first by Gatley et al. (1974), OMC2 is located 15' $(\sim 2 \mathrm{pc})$ north of the Orion nebula. It has a filamentary structure, elongated in the direction north-south, with active star formation concentrated in the central and densest region, shielded from the UV radiation from newly formed OB stars (Johnson et al. 1990). The mass of the cloud amounts to about $1500 M_{\odot}$ (Mezger et al. 1990). Several extensive studies have shown that OMC2 is a rich star-forming region, which harbors several young protostars, including several Class 0 candidates (Ali \& Depoy 1995; Chini et al. 1997; Lis et al. 1998; Johnstone \& Bally 1999; Reipurth et al. 1999). Observations of molecular lines have revealed several outflows emanating from the young protostars in the region. Many studies have focused on the outflows 
(e.g. Williams et al. 2003) and their impact on the cloud (Aso et al. 2000; Wu et al. 2005). Only a few of these studies, in contrast, have addressed the problem of the chemical structure of the forming stars in OMC2 (Johnstone et al. 2003).

Among the several protostars in OMC2, FIR4 stands out as the brightest submillimeter source (Mezger et al. 1990). Located almost at the center of the cloud, FIR4 is also a bright IRAS source and a VLA radio source (Reipurth et al. 1999). All these characteristics led Reipurth et al. (1999) to define FIR4 as "a bona fide Class 0 source". The FIR4 integrated luminosity was estimated to be about $400 L_{\odot}$ and the envelope mass is about $35 M_{\odot}$. Such values led to identifying FIR4 as anIM protostar (Johnstone et al. 2003). Because of its vicinity and its relatively bright molecular lines, FIR4 is an ideal source for a detailed study of the physical and chemical structure of an IM protostar. Existing dust continuum and molecular line observations point to an envelope with at least two components: a warm component with an average temperature of about $40 \mathrm{~K}$ and a colder component at about $15 \mathrm{~K}$ (Mezger et al. 1990; Johnstone et al. 2003). Jørgensen et al. (2006) modeled the $850 \mu \mathrm{m}$ SCUBA map towards this source to reconstruct its temperature and density profiles. Based on the observed $\mathrm{CO}$ and $\mathrm{H}_{2} \mathrm{CO}$ millimeter line emission, Jørgensen et al. (2006) conclude that the FIR4 envelope is illuminated by an external FUV field amounting to $1 \times 10^{4}$ times the interstellar FUV field. However, their interpretation suffers from some contradictions emphasized by the same authors. For example, such an intense FUV field would heat up the whole envelope to a temperature over $25 \mathrm{~K}$, the $\mathrm{CO}$ freezing temperature (Öberg et al. 2005), in contradiction to the measured average $\mathrm{CO}$ abundance, ten times lower than the canonical value, which would instead testify for a large CO-frozen region (Jørgensen et al. 2006). In addition, the maps of the fine structure lines of the $\mathrm{O}$ and $\mathrm{C}^{+}$atoms, together with the $\mathrm{CO}$ 1-0 line, led Herrmann et al. (1997) to conclude that the OMC2 region is illuminated by a FUV field 500 times the interstellar field.

Given this puzzling situation, we decided to again derive the temperature and density profiles of FIR4 by taking more data into account than those considered by Jørgensen et al. (2006) (Sect. 2). Using the derived dust temperature and density profiles, we then computed the gas temperature profile by equating the heating and cooling terms across the envelope (Sect. 3). As shown by several authors (e.g. Ceccarelli et al. 1996; Doty \& Neufeld 1997), the gas cooling in protostellar envelopes is dominated by the emission from the rotational lines of $\mathrm{CO}$ and, more important, $\mathrm{H}_{2} \mathrm{O}$ and the fine structure lines of OI. Actually, water is a key molecule in the gas thermal balance for two reasons. First, in the warm regions where the grain mantles sublimate, it is the most abundant molecule; second, given its relatively large dipole moment, water is a very powerful line emitter, hence a very powerful gas coolant. Given its major role in the prediction of the gas temperature profile, we discuss the dependence of the derived gas temperature on the assumed water abundance profile, which is poorly known. Not surprisingly, FIR4 is in fact one of the few sources where the full spectrum between 50 and $2000 \mathrm{GHz}$ is planned to be observed at high spectral resolution with the Heterodyne Instrument for the Far Infrared (HIFI) onboard Herschel (http: //herschel . esac. esa. int/), to be launched in 2009. HSO, and specifically the high resolution interferometer HIFI, will allow to observe the water lines in the 500 to $2000 \mathrm{GHz}$ range with unprecedented spectral and spatial resolution. Motivated by the Herschel mission, we report the predicted water line spectrum for the different assumed water abundance profiles, and discuss the observability by HIFI and PACS (Sect. 4). Section 5 concludes the article.

\section{Dust density and temperature profiles}

In this section, we derive the dust density and temperature profiles by modeling the 350,450 and $850 \mu \mathrm{m}$ maps of the region, plus the Spectral Energy Distribution (SED) from the millimeter to the Mid-Infrared (MIR) wavelength range. We first describe the observations we used in our analysis (Sect. 2.1), then the modeling (Sect. 2.2), and the result of the modeling (Sect. 2.3).

\subsection{Continuum emission: observational data}

In our analysis, we used the maps of the continuum emission at 850,450 , and $350 \mu \mathrm{m}$ obtained at JCMT and CSO, respectively. In addition, we take into account the Spectral Energy Distribution (SED) of FIR4 from 24 to $850 \mu \mathrm{m}$ obtained considering also the IRAS and Spitzer observations.

\section{a) 850,450 , and $350 \mu \mathrm{m}$ maps}

We retrieved the 450 and $850 \mu \mathrm{m}$ maps obtained by Johnstone \& Bally (1999) at the $15 \mathrm{~m}$ James Clerk Maxwell Telescope (JCMT) with the focal-plane instrument SCUBA (Submillimeter Common-User Bolometer Array). The spatial resolution of the maps is $7.5^{\prime \prime}$, and $14.8^{\prime \prime}$ at 450 , and $850 \mu \mathrm{m}$, respectively. The calibration uncertainty and noise levels are estimated by those authors $\lesssim 10 \%$ and $0.04 \mathrm{Jy}^{\text {beam }^{-1}}$ at $850 \mu \mathrm{m}$, and $\lesssim 30 \%$ and $0.3 \mathrm{Jy} \mathrm{beam}^{-1}$ at $450 \mu \mathrm{m}$, respectively. The $350 \mu \mathrm{m}$ map was obtained by Lis et al. (1998) at the $10.4 \mathrm{~m}$ telescope of the Caltech Submillimeter Observatory (CSO). The instrument used was the bolometer camera SHARC. The resolution of the map is $12^{\prime \prime}$. The calibration uncertainty has been evaluated $\sim 25 \%-30 \%$. The three maps are reported in Fig. 1. They show the envelope surrounding/forming FIR4, which extends for about $20^{\prime \prime}$, but also the presence of two sources: FIR3, 25" North, and FIR5, 25" South. To evaluate the continuum brightness profile of the FIR4 envelope, we averaged the continuum flux over ring at the same distance from the FIR4 center, excluding the regions contaminated by the presence of FIR3 and FIR5 (dashed regions in Fig. 1). The resulting brightness profiles are shown in Fig. 2. Note that in the analysis of the envelope emission (Sect. 2.2), we subtracted the cloud contribution, estimated to be $\sim 0.001$, $\sim 0.03$, and $\sim 0.05 \mathrm{Jy} \operatorname{arcsec}^{-2}$ at 850,450 , and $350 \mu \mathrm{m}$, respectively. Furthermore, to take into account that the SCUBA and SHARC maps were obtained with the chop throw of $65^{\prime \prime}$ and $90-120^{\prime \prime}$, respectively, we only considered the inner $60^{\prime \prime}$ in our analysis.

b) $S E D$

The SED points at 850,450 and $350 \mu \mathrm{m}$, shown in Fig. 2, were obtained by integrating the continuum emission over the envelope. We attributed an uncertainty of $\sim 30 \%$ to them to account for the uncertainty in the envelope size. We also considered the IRAS fluxes at 60 and $100 \mu \mathrm{m}$, respectively, extracted from the IRAS maps at these wavelengths. The fluxes were evaluated with the method previously employed for the maps at 850, 450, and $350 \mu \mathrm{m}$, namely excluding the same regions (dashed regions in Fig. 1) to limit the contamination by FIR3 and FIR5 and integrating over the rings. We also subtracted the cloud contribution, estimated to be $\sim 0.06$ and $\sim 0.07 \mathrm{Jy} \operatorname{arcsec}^{-2}$ at 60 and $100 \mu \mathrm{m}$, respectively. To account for the possible contamination of FIR3 and FIR5 due to the large beam of IRAS and the non-sphericity of the source, we took an uncertainty of $50 \%$ on the fluxes. Finally, we also considered the integrated flux at $24 \mu \mathrm{m}$ extracted from the Spitzer Space Telescope's Multiband Imaging Photometer (MIPS) maps. To this end, we retrieved the observations from the Spitzer Science archive (http:// ssc.spitzer.caltech.edu/archanaly/). The observations 


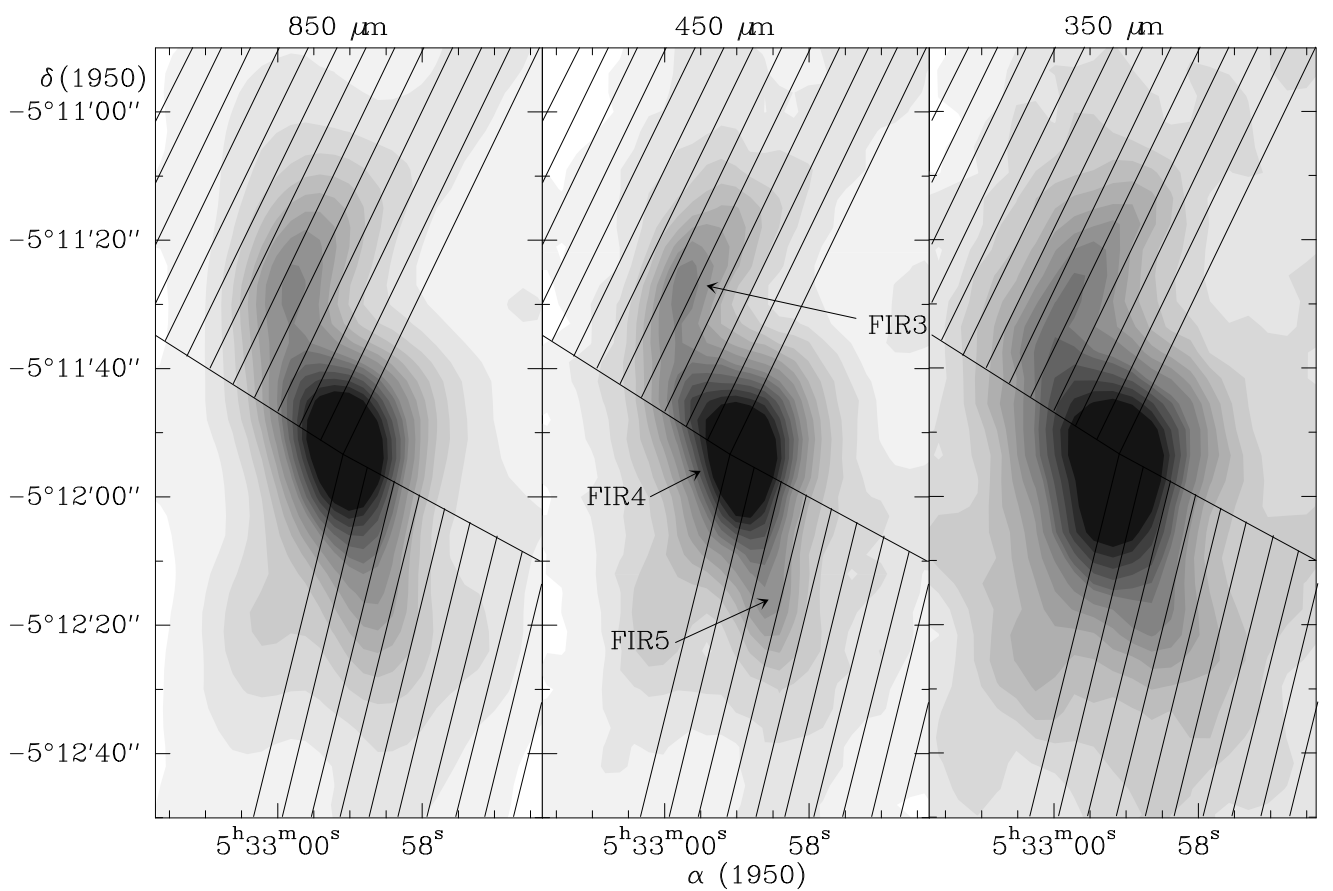

Fig. 1. Continuum emission maps around OMC2-FIR4 at $850 \mu \mathrm{m}$ (left panel), $450 \mu \mathrm{m}$ (middle panel), and $350 \mu \mathrm{m}$ (right panel). The contours mark the continuum flux from 5\% to $75 \%$ of the peak emission by steps of 5\%. The hatched regions have been excluded when computing the brightness profile of the FIR4 envelope (see text). The position of the three protostars in the regions, FIR3, FIR4, and FIR 5 are marked in the central panel figure.
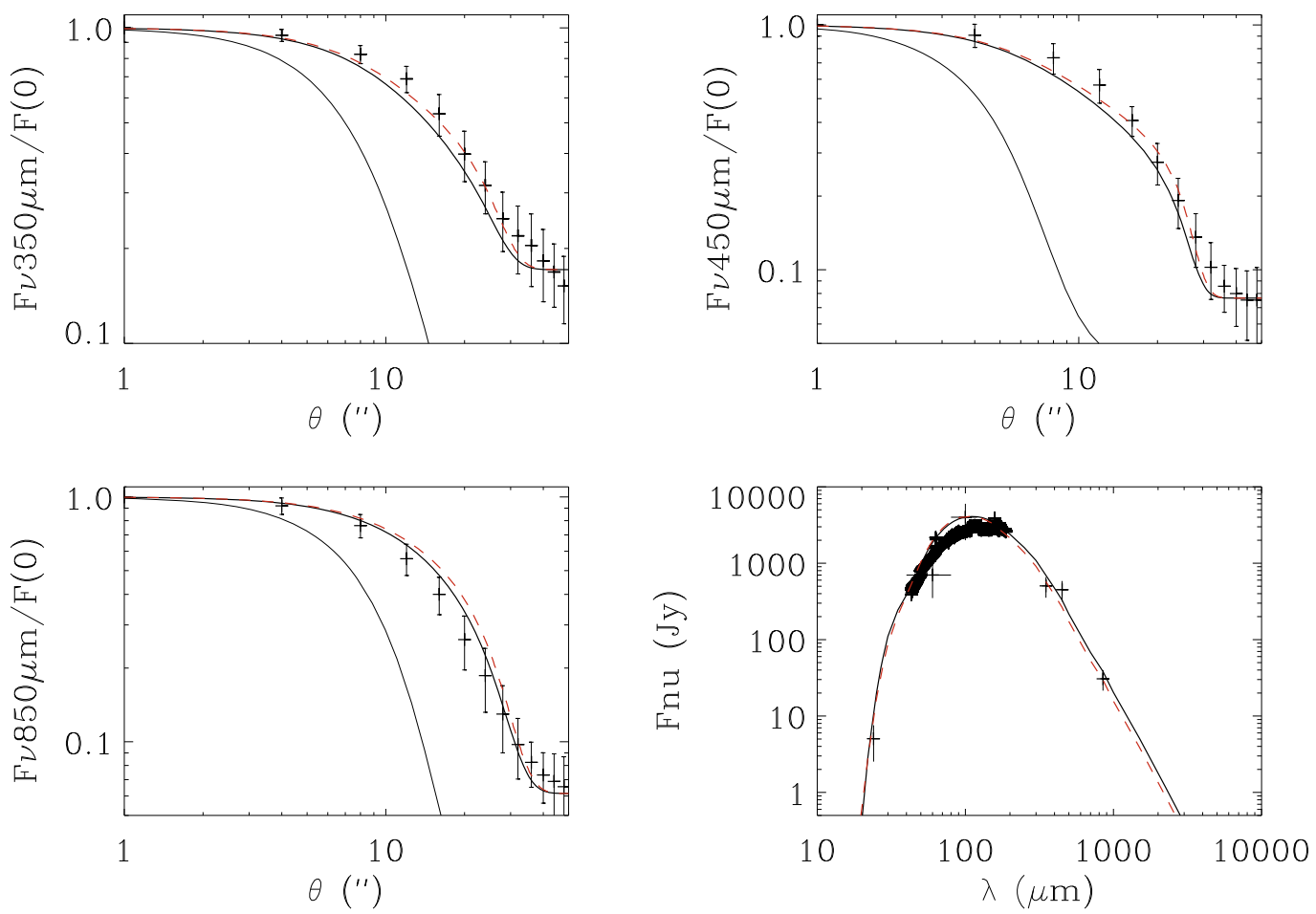

Fig. 2. Observed brightness profiles at 350 (upper left panel), 450 (upper right panel), $850 \mu \mathrm{m}$ (lower left panel), and SED (lower right panel). The curves report the best-fit obtained in the two cases $G_{0}=1$ (solid line) and 1000 (dashed line). The dashed-dotted lines represent the beam pattern of the telescope adopted at 350, 450, and $850 \mu \mathrm{m}$. Note that the SED plot reports the ISO-LWS spectrum between 45 and $200 \mu \mathrm{m}$ for completeness, although it has not been considered in the $\chi^{2}$ analysis, due to the relative larger calibration uncertainty compared to the IRAS data.

were obtained the 6th October 2006 as part of the program "Infrared Properties of Edge-on Young Stellar Object Disks" (AOR: 30765, PI: Karl Stapelfeldt). The data reduction was performed using the pipeline S16.0.1. The flux, $(5.0 \pm 2.5 \mathrm{Jy})$, in Fig. 2 was obtained by integration over a $15^{\prime \prime}$ radius.

\subsection{Continuum emission: modeling}

To derive the dust physical structure, namely the dust temperature and density profiles, we used the 1D radiative transfer code DUSTY (Ivezic \& Elitzur 1997). Briefly, giving as input 
the temperature of the central object and a dust density profile, DUSTY self-consistently computes the dust temperature profile and the dust emission. The comparison between the computed $350,450,850 \mu \mathrm{m}$ brightness profiles (namely the brightness versus the distance from the center of the envelope) and SED with the observed profiles and SED (see previous paragraph) makes it possible to constrain the density profile and, consequently, the temperature profile of the envelope.

To be compared with the observations, the theoretical emission is convolved with the beam pattern of the telescope. Following the recommendations for the relevant telescope, the beam is assumed to be a combination of Gaussian curves: at $850 \mu \mathrm{m}$, we use HPBWs of $14.5^{\prime \prime}, 60^{\prime \prime}$, and $120^{\prime \prime}$, with amplitudes of $0.976,0.022$, and 0.002 , respectively; at $450 \mu \mathrm{m}$, the HPBWs are $8^{\prime \prime}, 30^{\prime \prime}$, and $120^{\prime \prime}$ with amplitude ratios of 0.934 , 0.06 , and 0.006, respectively (Sandell \& Weintraub 2001); at $350 \mu \mathrm{m}$, we use HPBWs of $12^{\prime \prime}$ and $22^{\prime \prime}$, with amplitude ratios of $0.7,0.3$, respectively (Hunter et al. 1996).

We assumed that the envelope density follows a power-law,

$n(r)=n_{0} \times\left(\frac{r_{0}}{r}\right)^{\alpha}$

where the power-law index, $\alpha$, is a free parameter of the model, as well as the density $n_{0}$, the density at $r_{0}$. Besides, the envelope starts at a radius $R_{\text {in }}$ and extends up to $R_{\text {out }}$. Both $R_{\text {in }}$ and $R_{\text {out }}$ are free parameters of the model. The last input to DUSTY is the temperature of the central source, $T_{*}$, here assumed to be $5000 \mathrm{~K}$. We verified that the choice of this last parameter does not influence the results. Finally, the opacity of the dust as function of the wavelength is another parameter of DUSTY. Following numerous previous studies (van der Tak et al. 1999; Evans et al. 2001; Shirley et al. 2002; Young et al. 2003), we adopted the dust opacity calculated by Ossenkopf \& Henning (1994), specifically their OH5 dust model, which refers to grains coated by ice.

In summary, the output of DUSTY depends on $\alpha, n_{0}, R_{\text {in }}$, and $R_{\text {out }}$. In practice, the DUSTY input parameters are the power-law index, $\alpha$, the optical thickness at $100 \mu \mathrm{m}, \tau_{100}$, the ratio between the inner and outer radius, $Y\left(=R_{\text {out }} / R_{\text {in }}\right)$, and the temperature at the inner radius $T_{\text {in }}$. The optical thickness is, in turn, proportional to the dust column density, which depends on $n_{0}$ and the physical thickness of the envelope. Note that, since the beam of the available maps are relatively large ( $\geq 7.5^{\prime \prime}$ which corresponds to a linear length of $\geq 3300 \mathrm{AU}$ ), the inner region of the envelope is relatively unconstrained by the available observational data. In practice, we obtain a lower limit to $T_{\text {in }}$ of $300 \mathrm{~K}$ : any higher value would give similar results. Finally, as explained in Ivezic \& Elitzur (1997), DUSTY gives scaleless results (which makes it very powerful because the same grid of models can be applied to different sources). This means that, to compare the DUSTY output with actual observations, it is necessary to scale the output by the source bolometric luminosity $L_{\text {bol }}$ and the distance. Note that the bolometric luminosity is in fact estimated by integrating the emission over the full spectrum. By definition, this can only be done when the entire SED is known. This is exactly one of the outputs of the modeling, we re-evaluated the luminosity of FIR4 iteratively from the best-fit model, by minimizing the $\chi^{21}$. We anticipate here that the new value is 1000 rather than $400 L_{\odot}$, where we assumed the most recent estimation of the distance, namely $(437 \pm 19)$ pc (Hirota et al. 2007).

\footnotetext{
1 Note that, in the case of OMC2-FIR4, integrating the model SED gives the same results as integrating under the observed SED.
}

Table 1. Range of the input parameters to DUSTY covered in the present study.

\begin{tabular}{ll}
\hline \hline Parameter & Range \\
\hline$\alpha$ & $0.2-3.9$ \\
$Y$ & $100-2200$ \\
$\tau_{100}$ & $0.1-4.6$ \\
$T_{\text {in }}$ & $300 \mathrm{~K}$ \\
$T_{*}$ & $5000 \mathrm{~K}$ \\
\hline
\end{tabular}

The range of the $\alpha, Y$ and $\tau_{100}$ parameters is covered by increasing their respective value at each step of the grid by $20 \%$. Note that $T_{\text {in }}$ and $T_{*}$ are kept fixed because they do not influence the results (see text).

Table 2. best-fit parameters for the case $G_{0}=1$. Note that $\chi_{\text {red }}^{2}=\chi^{2} / v$ where $v$ is the number of degrees of freedom.

\begin{tabular}{lccccc}
\hline \hline Observation & $\alpha$ & $Y$ & $\tau_{100}$ & $\chi_{\text {red }}^{2}$ & $v$ \\
\hline $850 \mu$ m profile & 1.4 & 160 & - & 0.72 & 10 \\
$450 \mu$ m profile & 0.6 & 120 & - & 0.63 & 10 \\
$350 \mu$ m profile & 0.5 & 170 & - & 0.47 & 10 \\
All profiles & 0.6 & 120 & - & 1.24 & 36 \\
SED & - & - & 0.6 & 0.55 & 3 \\
\hline
\end{tabular}

The first line reports the best-fit obtained using only the $850 \mu$ m brightness profile; second line, using the $450 \mu \mathrm{m}$ brightness profile; third line, using the $350 \mu \mathrm{m}$ brightness profile; fourth line gives the best-fit using the three profiles; the last line gives the best-fit using the SED.

We ran a grid of models to cover the parameter space as reported in Table 1. The same grid of models were run for four values of the illuminating FUV field: $G_{0}=1,10,100$ and 1000 . In all cases, we used the Inter-Stellar Radiation Field (ISRF) constructed by Evans et al. (2001): combination of the radiation field introduced by Black (1994) with that of Draine (1978). Note that, since DUSTY makes the assumption of isotropic scattering, the computed MIR emission is largely overestimated in the presence of strong external fields (Elitzur, private communication). To solve this problem, we followed the suggestion by Young \& Evans (2005) to neglect the scattering, artificially putting it to zero. The best-fit model has been found by minimizing the $\chi^{2}$ with an iterated two-step procedure. First, we use the observed brightness profiles at 350,450 , and $850 \mu \mathrm{m}$ to constrain $\alpha$ and $Y$, assuming a value for $\tau_{100}$. Second, we constrain the optical thickness $\tau_{100}$ by comparing the computed and observed SED, assuming the $\alpha$ and $Y$ of the previous step. The new $\tau_{100}$ is used for a new iteration and so on. In practice, the iteration converges in two steps. This is because the normalized brightness profiles very weakly depend on $\tau_{100}$, while they very much depend on the sizes of the envelope and on the slope of the density profile (see also Jørgensen et al. 2002 and Schöier et al. 2002). In contrast, the optical thickness depends mostly on the absolute column density of the envelope, constrained by the SED.

\subsection{Results}

We run four grids of models, as discussed separately below: a) with a standard illumination FUV field $\left(G_{0}=1\right)$ and b) with a 10, 100, 1000 times enhanced field $\left(G_{0}=10,100,1000\right)$ (see Introduction). In paragraph c), we also discuss why larger $G_{0}$ was not considered, and in paragraph d) we summarize the results.

\section{a) $G_{0}=1$}

Table 2 presents the set of parameters $\alpha, Y$, and $\tau_{100}$, which reproduce the observations assuming $G_{0}=1$ better. Figure 2 

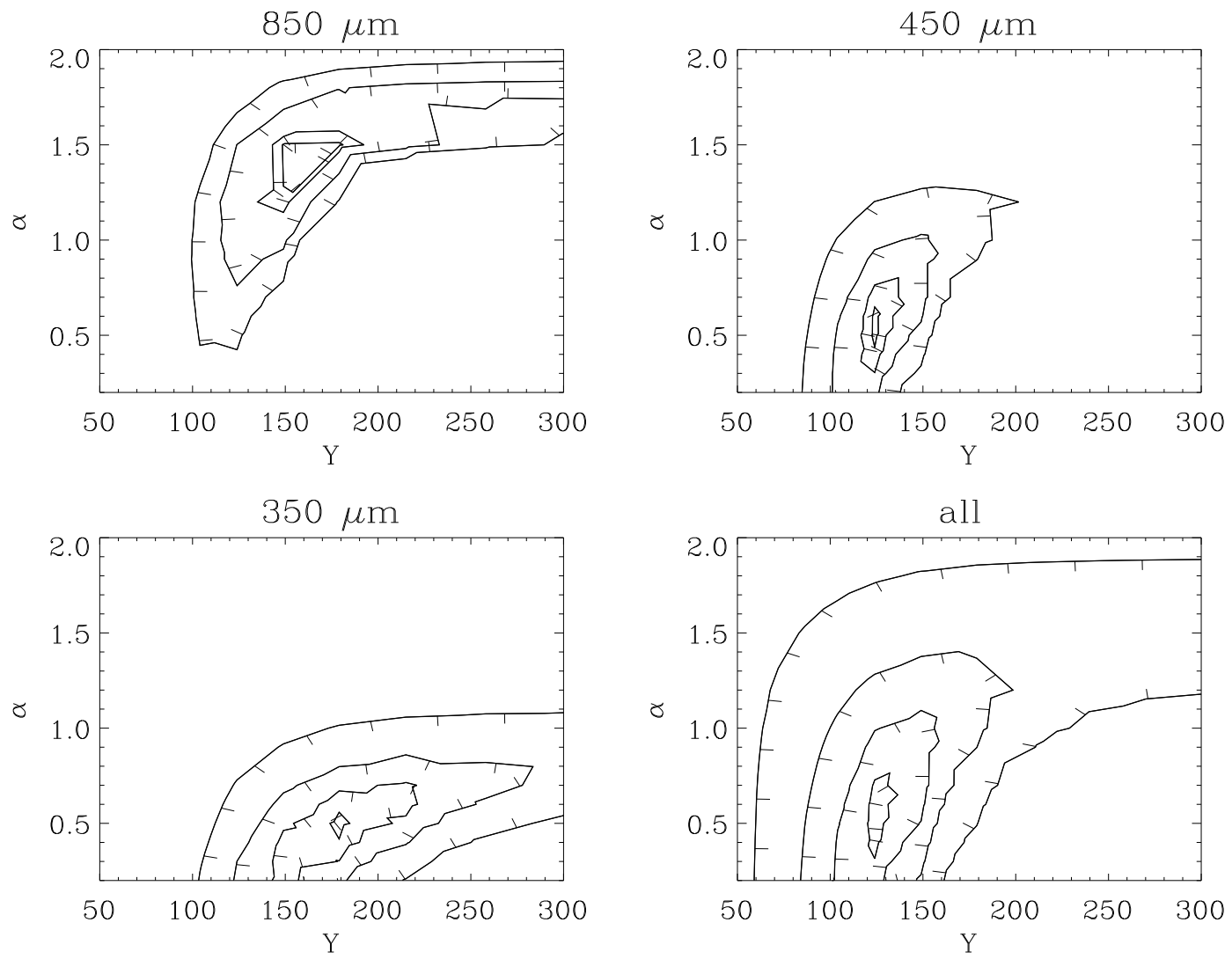

Fig. 3. $\chi_{\text {red }}^{2}$ contour plots $(Y, \alpha)$ for the models with $G_{0}=1$. In these computations, $\tau_{100}$ is equal to 0.6 . The contours show the loci of the $\chi_{\text {red }}^{2}$ values equal to $1.1,1.5,2.5$, and 5 times the minimum $\chi_{\text {red }}^{2}$. The upper left panel is obtained by comparing the model predictions with the $850 \mu \mathrm{m}$ brightness profile; the upper right panel refers to the $450 \mu \mathrm{m}$ profile; the lower left panel refers to the $350 \mu \mathrm{m}$ profile; the lower right panel makes use of the three profiles.

shows the relevant derived brightness profiles and SED against the observed ones. Figure 3 shows the $\chi^{2}$ contours plots obtained by considering separately the brightness profiles at 350,450 , and $850 \mu \mathrm{m}$, and by combining the three profiles. Figure 4 shows the $\chi^{2}$ dependence on the $\tau_{100}$ parameter.

The three $\chi_{350}^{2}, \chi_{450}^{2}$, and $\chi_{850}^{2}$ contour plots point to a value of $Y$ around 100-200. Conversely, the $\chi_{350}^{2}$ and $\chi_{450}^{2}$ contour plots constraint $\alpha$ to a lower value than 1 , around $0.5-0.6$, whereas the $\chi_{850}^{2}$ would instead indicate a higher value for $\alpha$, although the value 0.6 is still acceptable. Note that the solution found by Jørgensen et al. (2006) relies on the $850 \mu \mathrm{m}$ profile alone, so it gives a high $\alpha$ value, consistent with our $\chi_{850}^{2}$ plot. The $\chi_{\text {SED }}^{2}$ plot (Fig. 4) points to a value of $\tau_{100}$ of 0.6 . In minimizing the $\chi_{\text {SED }}^{2}$, we varied the source luminosity from 400 to $1500 L_{\odot}$. The best-fit is obtained for a source luminosity equal to $1000 L_{\odot}$.

b) $G_{0}=10,100,1000$

The best-fit values of $\alpha$ and $Y$ for cases of an enhanced illumination UV field are presented in Fig. 5. The first thing to notice is that the $\chi_{\text {all }}^{2}$ does not change appreciably for $G_{0}$ equal to 1,10 , 100, or 1000: the minimum $\chi_{\text {all red }}^{2}$ value is $1.24,1.23,1.19$, and 1.20 for $G_{0}=1,10,100$, and 1000 , respectively. In other words, the available continuum observations, both the profiles and the SED, cannot distinguish which of the four models is better. Furthermore, Fig. 5 shows that the $\chi_{\text {all }}^{2}$ contour plots point to the same $Y$ and $\alpha$ values. Similarly, the $\tau_{100}$ value is 0.6 for the four cases $G_{0}=1,10,100$, and 1000 . The situation is illustrated in Fig. 2, where the best fit predictions are compared to the

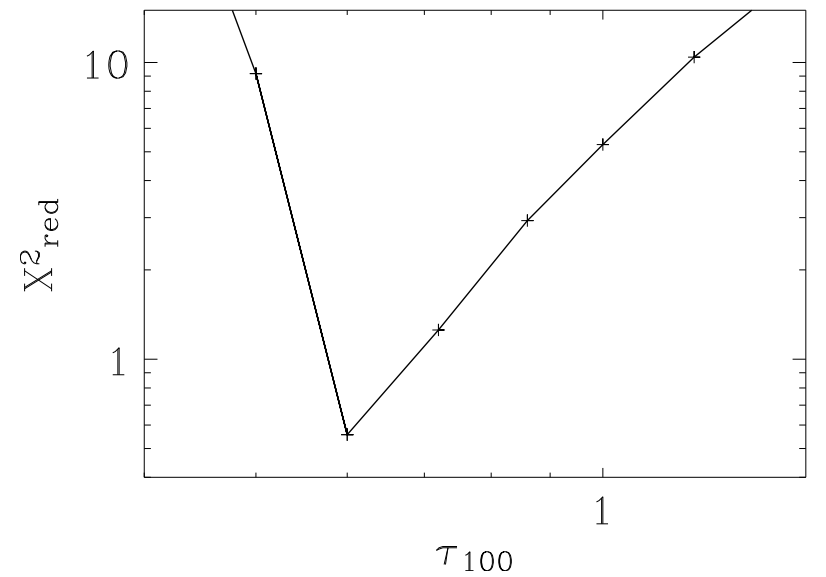

Fig. 4. $\chi_{\text {red }}^{2}$ versus $\tau_{100}$. In these computations, $Y$ is equal to 120 and $\alpha$ is equal to 0.6 .

observations for the two cases $G_{0}=1$ and 1000. Both models reproduce the observations fairly well, as is implicit in the best- $\chi^{2}$ similar values. However, note that the $G_{0}=1000$ case predicts slightly larger fluxes, due to the enhanced temperature at the border of the envelope.

\section{c) larger $G_{0}$}

We did not explore the case of larger $G_{0}$ in detail for three reasons. The first one is that previous line observations have shown 

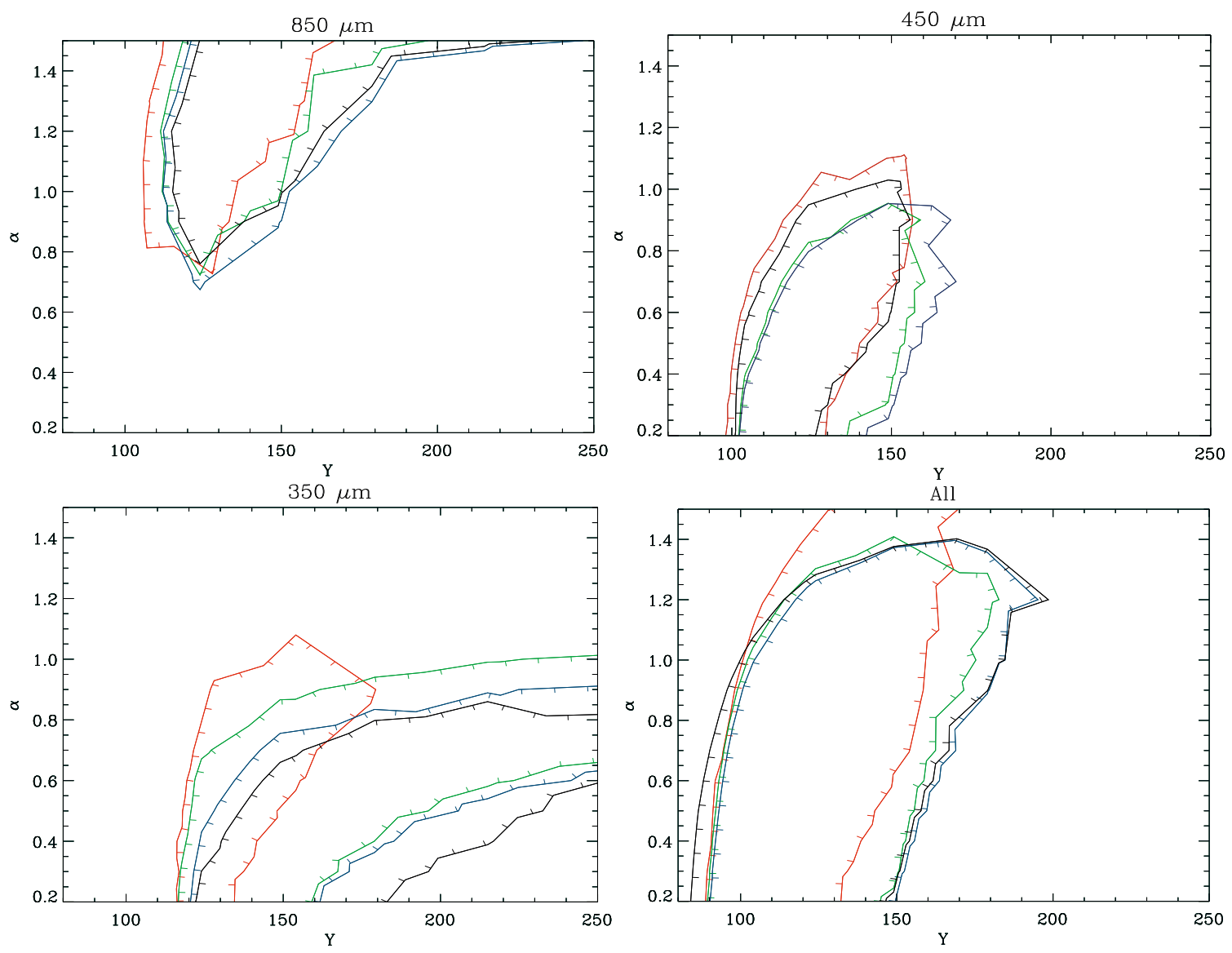

Fig. 5. $\chi_{\text {red }}^{2}$ contour plots $(\mathrm{Y}, \alpha)$ for the models with $G_{0}=1$ (black lines), 10 (blue lines), 100 (green lines), and 1000 (red lines). In these computations, $\tau_{100}$ is equal to 0.6 . The contours show the loci of the $\chi_{\text {red }}^{2}$ values equal to 2.5 times the minimum $\chi_{\text {red }}^{2}$. From top to bottom: $\chi^{2}$ contours of the $850 \mu \mathrm{m}, 450 \mu \mathrm{m}, 350 \mu \mathrm{m}$, and the three together.

that the FUV field in the OMC2 region is "only" 500 times the interstellar field. Indeed, Herrmann et al. (1997) mapped the OMC-2 cloud in the CII-157 $\mu \mathrm{m}$, OI-63 and $-146 \mu \mathrm{m}$ lines with the spectrometer FIFI onboard the Kuiper Airborne Observatory. They detected extended emission associated with the Photo-Dissociation Region (PDR) enveloping the whole OMC-2 molecular cloud. These authors concluded that OMC-2 is illuminated by a FUV field whose intensity is $G_{0} \sim 500$. Note that this is the FUV field impinging on the cloud, then the effective $G_{0}$ seen by the FIR4 envelope is probably lower than this. The second reason is that varying $G_{0}$ from 1 to $10^{3}$ does not improve the $\chi^{2}$ value. The third reason is that the $G_{0}=10^{4}$ case suffers from severe convergence problems, and it was not possible to compute enough runs for a meaningful $\chi^{2}$ analysis.

\section{d) Summary of the adopted solution}

Table 3 summarizes the value of the best fit parameters, obtained by considering all the profiles and the SED $\chi^{2}$ contour plots and assuming the $G_{0}=1$ case. Some relevant physical quantities are quoted in the same table. Figure 6 shows the dust density and temperature profiles of the best-fit models with $G_{0}=1$ and 1000 , respectively. Note that the dust temperature in the skin of the envelope is higher by $\sim 20-30 \mathrm{~K}$ in the case $G_{0}=1000$ with respect to the $G_{0}=1$ case. This increase concerns a relatively small region, of a few thousand AU. Jørgensen et al. (2006) found a larger warm region, of about $10^{4} \mathrm{AU}$, because of the steeper adopted density distribution $(\alpha=2)$ : in this case, the FUV photons can penetrate deeper into the envelope.

\section{Gas temperature profile}

\subsection{Model description}

Ceccarelli et al. (1996), Doty \& Neufeld (1997), and Maret et al. (2002) have shown that the gas is thermally decoupled from dust in the inner regions of low- and high-mass protostellar envelopes. The reason for that is the large water abundance in the gas phase caused by the sublimation of the grain mantles. The same phenomenon may occur in the envelopes of IM protostars, so we explicitly computed the gas temperature profile of the envelope surrounding FIR4. For that we explicitly computed the equilibrium temperature by equating the gas cooling and heating terms at each radius. Following the method described in Ceccarelli et al. (1996), we considered heating from the gas compression (due to the collapse), dust-gas collisions, and photopumping of $\mathrm{H}_{2} \mathrm{O}$ and $\mathrm{CO}$ molecules by the IR photons emitted by the warm dust close to the center ${ }^{2}$. The cooling is mainly due to rotational lines from $\mathrm{H}_{2} \mathrm{O}$ and $\mathrm{CO}$, plus the fine structure lines from $\mathrm{O}$. Therefore, the gas temperature depends on the abundance of these three species. In practice, though, only the water abundance is a real parameter of the model, because the $\mathrm{CO}$ and $\mathrm{O}$ lines are optically thick in the range of the $\mathrm{CO}$ and $\mathrm{O}$ abundances typical of protostellar envelopes. For this reason, we computed various cases for the water abundance, since it is generally poorly constrained in protostellar envelopes and totally

\footnotetext{
${ }^{2}$ Cosmic rays ionization is a minor heating term in the protostellar envelopes.
} 
Table 3. Summary of the dust radiative transfer analysis of OMC2FIR4.

\begin{tabular}{lr}
\hline \hline \multicolumn{2}{c}{ Fixed input parameters } \\
\hline Distance, $d$ & $437 \mathrm{pc}$ \\
Stellar temperature, $T_{\star}$ & $5000 \mathrm{~K}$ \\
Dust temperature at $r_{\mathrm{i}}, T_{\mathrm{d}}\left(r_{\mathrm{i}}\right)$ & $300 \mathrm{~K}$ \\
Dust opacity $(\mathrm{OH} 5)$ at $100 \mu \mathrm{m}, \kappa_{100}$ & $86.5 \mathrm{~cm}^{2} \mathrm{~g}^{-1}$ \\
\hline \multicolumn{2}{c}{ Best fit parameters } \\
\hline Luminosity, $L$ & $1000 L_{\odot}$ \\
Dust optical depth at $100 \mu \mathrm{m}, \tau_{100}$ & 0.6 \\
Density power-law index, $\alpha$ & 0.6 \\
Envelope thickness, $r_{\text {out }} / r_{\mathrm{i}}$ & 120 \\
\hline \multicolumn{2}{c}{ Physical quantities } \\
\hline Inner envelope radius, $r_{\text {in }}$ & $100 \mathrm{AU}$ \\
Outer envelope radius, $r_{\text {out }}$ & $12000 \mathrm{AU}$ \\
Radius at $\mathrm{T}_{\text {dust }}=100 \mathrm{~K}, r_{100 \mathrm{~K}}$ & $440 \mathrm{AU}$ \\
$\mathrm{H}_{2}$ density at $r_{100 \mathrm{~K}}, n_{0}$ & $4.3 \times 10^{6} \mathrm{~cm}^{-3}$ \\
Envelope mass, $M_{\text {env }}$ & $30 M_{\odot}$ \\
\hline
\end{tabular}

The first part lists the fixed input parameters, the second part reports the best-fit parameters, and some relevant physical quantities corresponding to the best-fit model are reported in the third part.

Table 4. The different run models.

\begin{tabular}{cccccc}
\hline Model & $X(\mathrm{CO})$ & $X(\mathrm{O})$ & $X\left(\mathrm{H}_{2} \mathrm{O}\right)_{\text {out }}$ & $X\left(\mathrm{H}_{2} \mathrm{O}\right)_{\text {in }}$ & $G_{0}$ \\
\hline $1^{a}$ & $1 \times 10^{-4}$ & $5 \times 10^{-4}$ & $1 \times 10^{-8}$ & $1 \times 10^{-5}$ & 1 \\
2 & $1 \times 10^{-4}$ & $5 \times 10^{-4}$ & $1 \times 10^{-8}$ & $1 \times 10^{-6}$ & 1 \\
3 & $1 \times 10^{-4}$ & $5 \times 10^{-4}$ & $1 \times 10^{-8}$ & $1 \times 10^{-4}$ & 1 \\
4 & $1 \times 10^{-4}$ & $5 \times 10^{-4}$ & $1 \times 10^{-9}$ & $1 \times 10^{-5}$ & 1 \\
5 & $1 \times 10^{-4}$ & $5 \times 10^{-4}$ & $1 \times 10^{-7}$ & $1 \times 10^{-5}$ & 1 \\
6 & $1 \times 10^{-4}$ & $5 \times 10^{-4}$ & $1 \times 10^{-7}$ & $1 \times 10^{-4}$ & 1 \\
7 & $1 \times 10^{-4}$ & $5 \times 10^{-4}$ & $1 \times 10^{-8}$ & $1 \times 10^{-5}$ & 1000 \\
\hline
\end{tabular}

Columns 2 to 5 report the adopted abundances of the main gas coolants: $X(\mathrm{CO}), X(\mathrm{O})$ and the $\mathrm{H}_{2} \mathrm{O}$ abundance in the $T \geq 100 \mathrm{~K}$ region $X\left(\mathrm{H}_{2} \mathrm{O}\right)_{\text {in }}$ and outer region $X\left(\mathrm{H}_{2} \mathrm{O}\right)_{\text {out }}$. Column 5 reports the FUV illuminating field $G_{0}$. Note: ${ }^{a}$ Model 1 is the reference for the studies of the water line spectrum presented in Sect. 4.

unconstrained in FIR4 (see Sect. 4). We adopted a step function for the water abundance profile to simulate the jump caused by the ice sublimation. The jump is assumed to occur at $100 \mathrm{~K}$. We considered the $\mathrm{H}_{2} \mathrm{O}$ abundance (with respect to $\left.\mathrm{H}_{2}\right) X\left(\mathrm{H}_{2} \mathrm{O}\right)_{\text {out }}$ in the outer envelope, where $T \leq 100 \mathrm{~K}$, equal to $10^{-7}, 10^{-8}$, and $10^{-9}$. We also considered three cases for the abundance in the inner region $X\left(\mathrm{H}_{2} \mathrm{O}\right)_{\text {in }}, 10^{-4}, 10^{-5}$, and $10^{-6}$. Finally, we studied the case with $G_{0}=1000$. The run parameters are summarized in Table 4.

To compute the cooling from the lines we used the code described in Ceccarelli et al. (1996, 2003) and Parise et al. (2005). The same code has been used in several earlier studies, whose results have been substantially confirmed by other groups (e.g. the analysis on IRAS16293-2422 by Schöier et al. 2002). Briefly, the code is based on the escape probability formalism in the presence of warm dust (see Takahashi et al. 1983), where the escape probability $\beta$ is computed at each point by integrating the line and dust absorption over the solid angle $\Omega$ as

$\beta=\frac{k_{\mathrm{d}}}{k_{\mathrm{L}}+k_{\mathrm{d}}}+\frac{k_{\mathrm{L}}}{\left(k_{\mathrm{L}}+k_{\mathrm{d}}\right)^{2}} \int \mathrm{d} \mu \frac{1-\exp \left[-\left(k_{\mathrm{L}}+k_{\mathrm{d}}\right) \Delta L_{\mathrm{th}}\right]}{\Delta L_{\mathrm{th}}}$
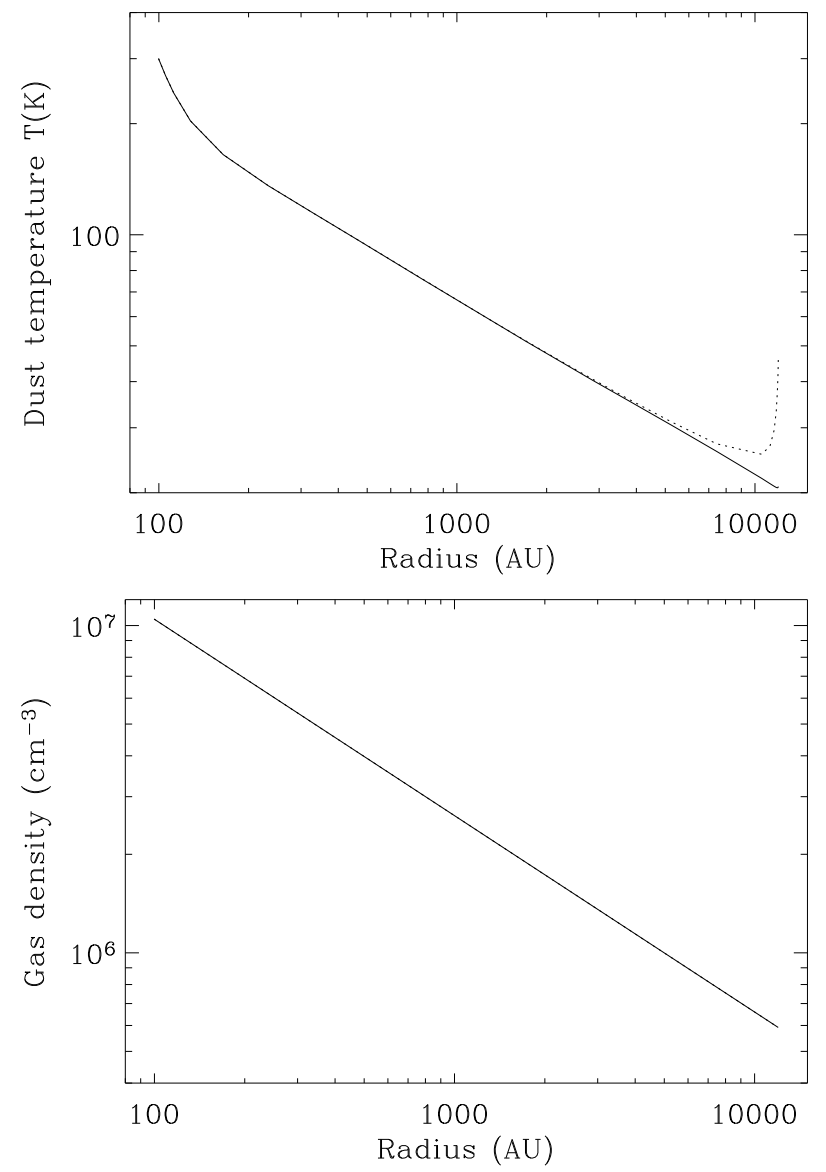

Fig. 6. Dust temperature (upper panel) and $\mathrm{H}_{2}$ density (lower panel) profiles from the best-fit obtained in the two cases $G_{0}=1$ and 1000 . The plain line and the dotted line represent the cases $G_{0}=1$ and 1000, respectively.

where $k_{\mathrm{L}}$ and $k_{\mathrm{d}}$ are the line and dust absorption coefficients, respectively, and $\Delta L_{\mathrm{th}}$ is the line trapping region, given by the following expressions:

$\Delta L_{\mathrm{th}}=2 \Delta v_{\mathrm{th}}\left(\frac{v}{r}\left|1-\frac{3}{2} \mu^{2}\right|\right)^{-1}$

in the infalling region of the envelope (where $\operatorname{arcos}(\mu)$ is the angle with the radial outward direction), and

$\Delta L_{\mathrm{th}}=r\left(1-\frac{r}{R_{\mathrm{env}}}\right)$

in the static region (where $R_{\mathrm{env}}$ is the envelope radius). In the present calculations, we assumed that the entire envelope is collapsing in free-fall towards a central object of $2 M_{\odot}$. In practice, the photons emitted by the dust can be absorbed by the gas and can pump the levels of the water molecules. This, indeed, is an important factor in the population of the water levels, and for the highest energy levels, even the dominant one (Sect. 4). In addition, $\mathrm{H}_{2} \mathrm{O}$ and $\mathrm{CO}$ molecules can be pumped by absorption of the NIR photons emitted by the innermost warm dust. Since the densities and temperatures of the regions of the envelope targeted by this study are not enough to populate the levels at the vibrational states, the effect of the NIR photons is an extra heating of the gas, as described in the Ceccarelli et al. (1996) article. Note that the code takes the dust with temperatures up to $1500 \mathrm{~K}$ into account, by following the algorithm described in Ceccarelli et al. (1996). 
For the collisional coefficients of water with hydrogen molecules, we used the data by Faure et al. (2007) available for the temperature range $20-2000 \mathrm{~K}$. This data set includes quasiclassical results for the highest rates (those over $10^{-12} \mathrm{~cm}^{3} \mathrm{~s}^{-1}$ ) and quantum scaled $\mathrm{H}_{2} \mathrm{O}-\mathrm{He}$ results for the lowest rates. Recent quantum calculations on ortho- $\mathrm{H}_{2} \mathrm{O}$ by Dubernet and co-workers have shown that the quasi-classical rates can be in error by as much as a factor of 100 but that, in general, they are accurate to within a factor of 1-3 (Dubernet et al. 2009). It should be noted that the rates of Faure et al. (2007) are currently the only complete and consistent set of data for both ortho- and para$\mathrm{H}_{2} \mathrm{O}$ colliding with $\mathrm{H}_{2}$. We also note that these rates have been recently extrapolated to cover energy levels and temperatures up to $5000 \mathrm{~K}$ (Faure \& Josselin 2008). Since the ortho-to-para conversion process of $\mathrm{H}_{2}$ is chemical rather than radiative, the ortho-to-para ratio $\mathrm{H}_{2}$ OPR is highly uncertain in the interstellar medium. Here we assume that in warmer gas it is in Local Thermal Equilibrium (LTE) and, therefore, follows the Boltzmann distribution:

$O P R=\frac{\left(2 I_{\mathrm{o}}+1\right) \Sigma(2 J+1) \exp \left(-\frac{E_{\mathrm{o}}(J)}{k T}\right)}{\left(2 I_{\mathrm{p}}+1\right) \Sigma(2 J+1) \exp \left(-\frac{E_{\mathrm{p}}(J)}{k T}\right)}$

where $I_{\mathrm{O}}$ and $I_{\mathrm{p}}$ are the total nuclear spin, corresponding to whether the hydrogen nuclear spins are parallel $\left(I_{\mathrm{o}}=1, \uparrow \uparrow\right)$ or anti-parallel $\left(I_{\mathrm{p}}=0, \uparrow \downarrow\right)$. The sum in the numerator and denominator extends over all ortho and para levels $J$, respectively. Similarly to $\mathrm{H}_{2}$, water comes in the ortho and para forms. In this case, since the water is the dominant gas coolant only in the regions where the dust temperature exceeds $100 \mathrm{~K}$, we assumed OPR equal to 3, strictly valid for gas temperatures over $60 \mathrm{~K}$. Since the water lines are optically thick, the cooling depends on the velocity field, assumed to be that of an envelope collapsing in free-fall towards a central object of $2 M_{\odot}$ (see above). We checked the influence of our results against this assumption, running a case with a constant velocity field of $0.5 \mathrm{~km} \mathrm{~s}^{-1}$. The difference in the gas temperature between the two cases never exceeds $10 \mathrm{~K}$.

\subsection{Results}

Figure 7 shows the computed gas temperature profile obtained with different values of $X\left(\mathrm{H}_{2} \mathrm{O}\right)_{\text {in }}$ in the case $G_{0}=1$. Figure 8 shows the different contributions to the heating and cooling rates. Similar to what had been found in low-mass protostars (Ceccarelli et al. 1996, 2000; Maret et al. 2002), the gas temperature tracks the dust temperature in the outer envelope, while gas and dust are decoupled in the inner part of envelope, where the icy grain mantles sublimate. The heating is dominated by compression of the collapsing gas across the entire envelope, even though the dust-gas collisions becomes comparable to the compression heating in the inner envelope. Although important in the very inner regions, the $\mathrm{H}_{2} \mathrm{O}$ photo-pumping never dominates the heating in contrarst to what happens in the studied lowmass protostars. The cooling, on the other hand, is dominated by $\mathrm{H}_{2} \mathrm{O}$ line emission in the inner envelope, by the $\mathrm{OI}$ line emission in the intermediate region, and by the $\mathrm{CO}$ in the outermost regions of the envelope. Note that the increased water abundance causes an increased cooling of the gas, which brings the equilibrium gas temperature to lower values than the dust temperature. This phenomenon, already predicted in low-mass protostars, is much more marked in the FIR4 case, leading to more than a $100 \%$ difference (with respect to the gas temperature) in the dust and gas temperatures for the case of the highest water abundance

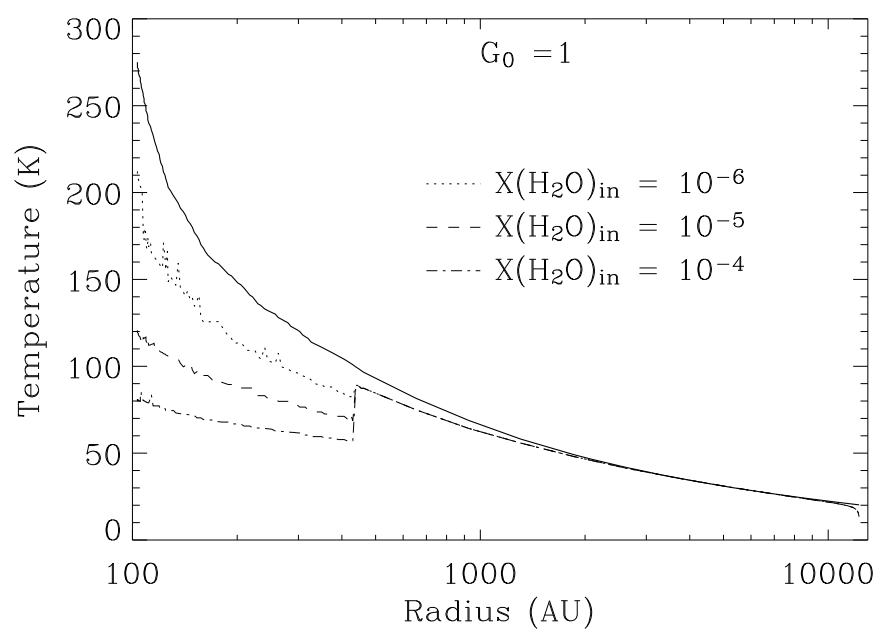

Fig. 7. The gas temperature profile of the collapsing envelope of OMC2FIR4. The different curves refer to different values of the inner envelope water abundance $X\left(\mathrm{H}_{2} \mathrm{O}\right)_{\text {in }}: 1 \times 10^{-6}$ (dotted), $1 \times 10^{-5}$ (dashed), and $1 \times$ $10^{-4}$ (dotted-dashed), respectively. In these computations, $X\left(\mathrm{H}_{2} \mathrm{O}\right)_{\text {out }}$ is $1 \times 10^{-8}$ and $G_{0}=1$. The solid line refers to the dust temperature profile.
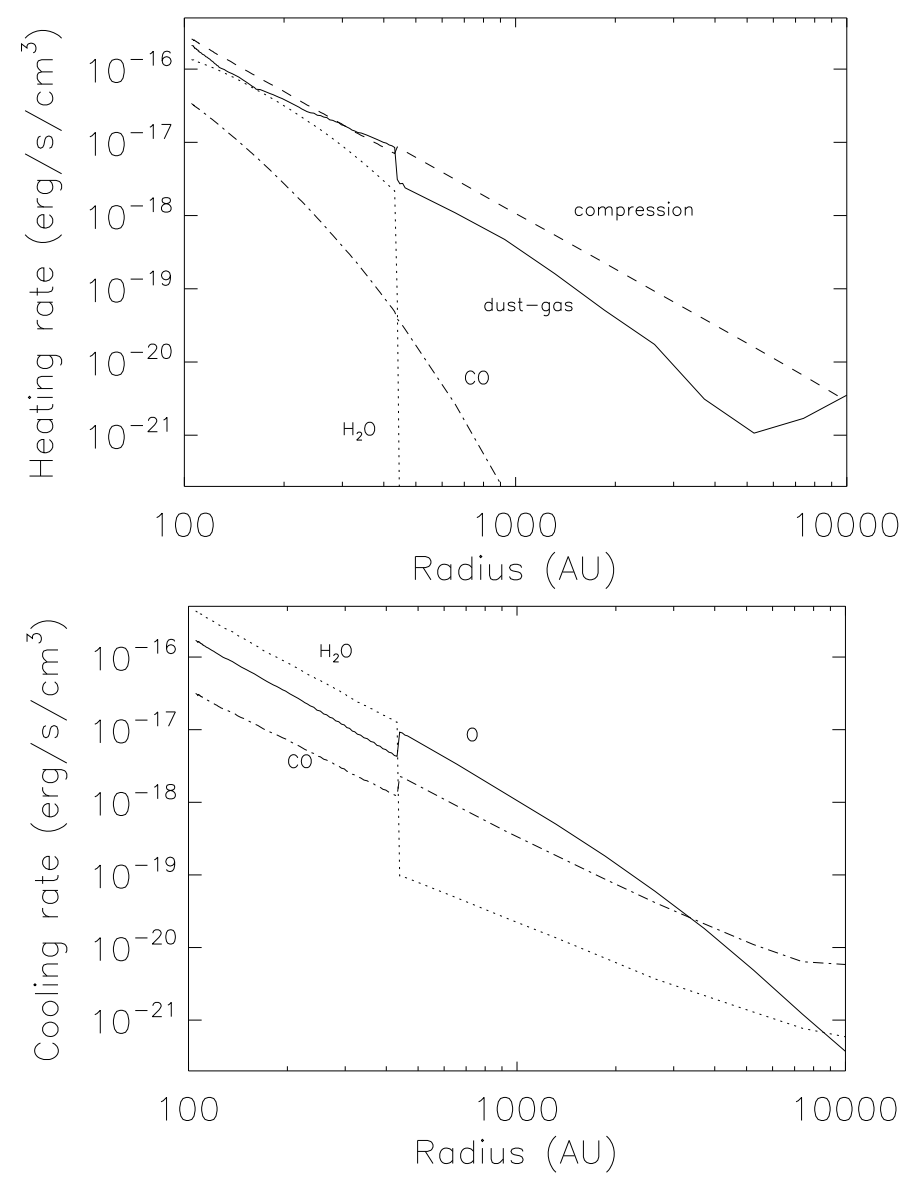

Fig. 8. Heating (top panel) and cooling (bottom panel) rates as function of the radius, computed assuming that the inner $\mathrm{H}_{2} \mathrm{O}$ abundance is equal to $1 \times 10^{-5}$ while the outer abundance is $1 \times 10^{-8}$.

$\left(1 \times 10^{-4}\right)$. For example, the dust temperature is $300 \mathrm{~K}$ at $100 \mathrm{AU}$, whereas the gas temperature varies from 200 to $80 \mathrm{~K}$ depending on the assumed $X\left(\mathrm{H}_{2} \mathrm{O}\right)_{\text {in }}, 1 \times 10^{-6}$ and $1 \times 10^{-4}$, respectively. The phenomenon is more marked in FIR4 than in the studied low-mass protostars because of the relatively lower density of the region where the icy grain mantles sublimate in FIR 4 rather 


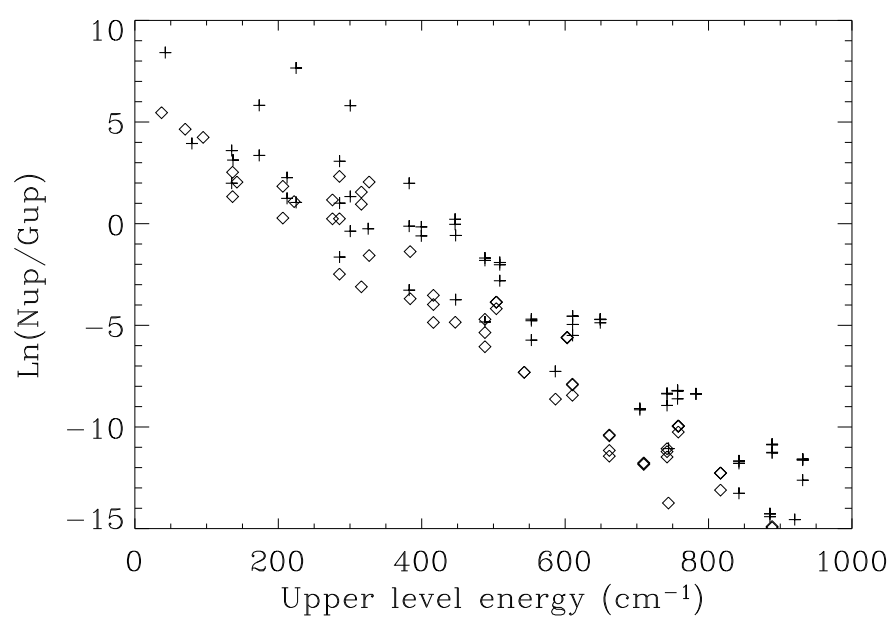

Fig. 9. Synthetic rotational diagram derived from the water line emission using the reference model (Model 1, Table 4) integrated over the whole envelope. Crosses trace the ortho and diamonds the para water.

than in the low-mass protostars, or, in other words, because the FIR4 envelope is warmer. Note that we obtain similar results also for larger illuminating FUV fields.

We emphasize that this result is a consequence of the derived shallow dependence of the density distribution, which is constrained from the fit of the maps. The dependence is strictly valid only on scales larger than the smaller telescope beam, namely $8^{\prime \prime}$ (equivalent to a radius of about $1700 \mathrm{AU}$ ), and the SED fit only gives the total column density, which, coupled with the density dependence on the radius (constrained by the maps), constrains the density on these scales. While we cannot exclude the presence of a denser compact object hidden by the envelope, it seems unlikely that the envelope density gradient increases inwards, because this would be unphysical.

Clearly, the water abundance in the inner region of FIR4 will have a strong impact not only on the emerging water spectrum but also on the emerging line spectrum of any molecule (abundant in the inner region), and has to be correctly taken into account to give reliable molecular abundances. Conversely, given the strong effect, in principle appropriate multiline observations of any molecule will be able to constrain the inner region water abundance and the present model predictions. Note that varying the outer abundance $X\left(\mathrm{H}_{2} \mathrm{O}\right)_{\text {out }}$ has no effect on the gas temperature, as in the outer region the cooling is dominated by the $\mathrm{CO}$ and $\mathrm{O}$ lines.

\section{Predicted water line spectrum}

\subsection{Reference model}

Here we report and discuss the predicted spectrum of our reference model. The next paragraph will discuss how it depends on the parameters of the model. We adopted Model 1 of Table 4 as reference model. We first discuss the general water line spectrum by means of the synthetic rotational diagram, and then discuss the specific predictions for the two spectrometers onboard Herschel: HIFI and PACS.

Figure 9 shows the synthetic rotational diagram derived from the line emission integrated over the whole envelope. As expected, the theoretical points do not lie on a compact and straight line, reflecting the different line optical depths, the gradients in density and temperature of the envelope, and non-LTE effects. An illuminating example is represented by the fundamental transitions of the ortho and para water lines at 557 and 1113 $\mathrm{GHz}$, respectively. We discuss these two lines in detail because, first, they will certainly be important observational diagnostics and, second, they offer a good pedagogical case. The situation is illustrated in Fig. 10, where we report the profile of the emission of the ortho and para $\mathrm{H}_{2}^{16} \mathrm{O}$ and $\mathrm{H}_{2}^{18} \mathrm{O}$ fundamental lines as a function of the radius. Figure 11, with the beta escape probability as function of the radius for the two fundamental $\mathrm{H}_{2}^{16} \mathrm{O}$ lines, also helps for interpreting the emerging line fluxes for the two lines. The ortho- $\mathrm{H}_{2}^{16} \mathrm{O}$ fundamental line emission (Fig. 10) peaks at the border of the envelope, and it decreases inwards because of the decreasing emitting volume. The para- $\mathrm{H}_{2} \mathrm{O}$ fundamental line shows approximately the same behavior. If the lines were optically thin and LTE populated, the expected flux ratio of the para over ortho fundamental line would be between 3 and 4 for a temperature between 50 and $200 \mathrm{~K}$. Any departure from this value originates from a combination of line opacity and non-LTE effects. In the outer region, the ratio is lower than 1: the para- $\mathrm{H}_{2} \mathrm{O}$ line is optically thin, whereas the ortho- $\mathrm{H}_{2} \mathrm{O}$ lines is moderately optically thick (Fig. 11). Therefore, the much lower emission of the para- $\mathrm{H}_{2} \mathrm{O}$ line than the ortho-line is due to the non-LTE population effect, which is more accentuated in the para- $\mathrm{H}_{2} \mathrm{O}$ line. The situation is reversed in the inner region, where ices sublimate: the para- $\mathrm{H}_{2} \mathrm{O}$ fundamental line becomes about ten times brighter than the ortho- $\mathrm{H}_{2} \mathrm{O}$ fundamental line because of the line opacity, which is much greater in the ortho- $\mathrm{H}_{2} \mathrm{O}$ line than in the para- $\mathrm{H}_{2} \mathrm{O}$ line (Fig. 11). In fact, the increase in the water abundance by a factor 1000 gives rise to a jump in the line emission by a factor 3 in the ortho- $\mathrm{H}_{2} \mathrm{O}$ line and 30 in the para- $\mathrm{H}_{2} \mathrm{O}$ line, and this can only stem from the higher opacity of the ortho- $\mathrm{H}_{2} \mathrm{O}$ line as the excitation conditions do not change when ices sublimate. In summary, the emission from the water lines comes, in principle, from a rather complex combination of line opacity, non-LTE effects and emitting volume (namely temperature and density gradient). Evidently, the intensity ratio of lines from the $\mathrm{H}_{2}^{16} \mathrm{O}$ and $\mathrm{H}_{2}^{18} \mathrm{O}$ isotopologues is not enough to obtain the "opacity" of the line, as it is a combination of the penetration of the line and the opacity itself.

Table 5 lists the predicted water line fluxes for the two spectrometers on board Herschel: HIFI and PACS. Note that, in both cases, we computed the signal after convolving the theoretical line intensity map with the instrument beam which vary from $39^{\prime \prime}$ to $13^{\prime \prime}$ with the frequency varying from $500 \mathrm{GHz}$ to $2000 \mathrm{GHz}$ (HIFI frequency range) and from 13" to $5^{\prime \prime}$ for wavelengths from $210 \mu \mathrm{m}$ and $60 \mu \mathrm{m}$ (PACS wavelength range).

Based on the (preliminary) sensitivities reported on the Herschel Observation Planning Tool HSpot: http:// herschel.esac.esa.int/Tools.shtml), several ortho and para lines are predicted to be detectable by the two Herschel spectrometers: about a dozen in the HIFI frequency range and twice more in the PACS wavelength range. The $\mathrm{H}_{2}^{18} \mathrm{O}$ ortho and para lines are also predicted to be detectable by HIFI, and 100 and 20 times fainter than the respective lines of the $\mathrm{H}_{2}^{16} \mathrm{O}$. We note the counter-intuitive result: the para- $\mathrm{H}_{2}^{16} \mathrm{O}$ line seems to be more optically thick than the ortho- $\mathrm{H}_{2}^{16} \mathrm{O}$ line! As explained above, this is not the case, of course: the line intensity ratio (from which the line optical depth is usually derived) comes from the combination of optical depth and excitation (non-LTE) effects, and the final result is not easily predictable. In our reference model, no observable line is predicted to be in absorption. 

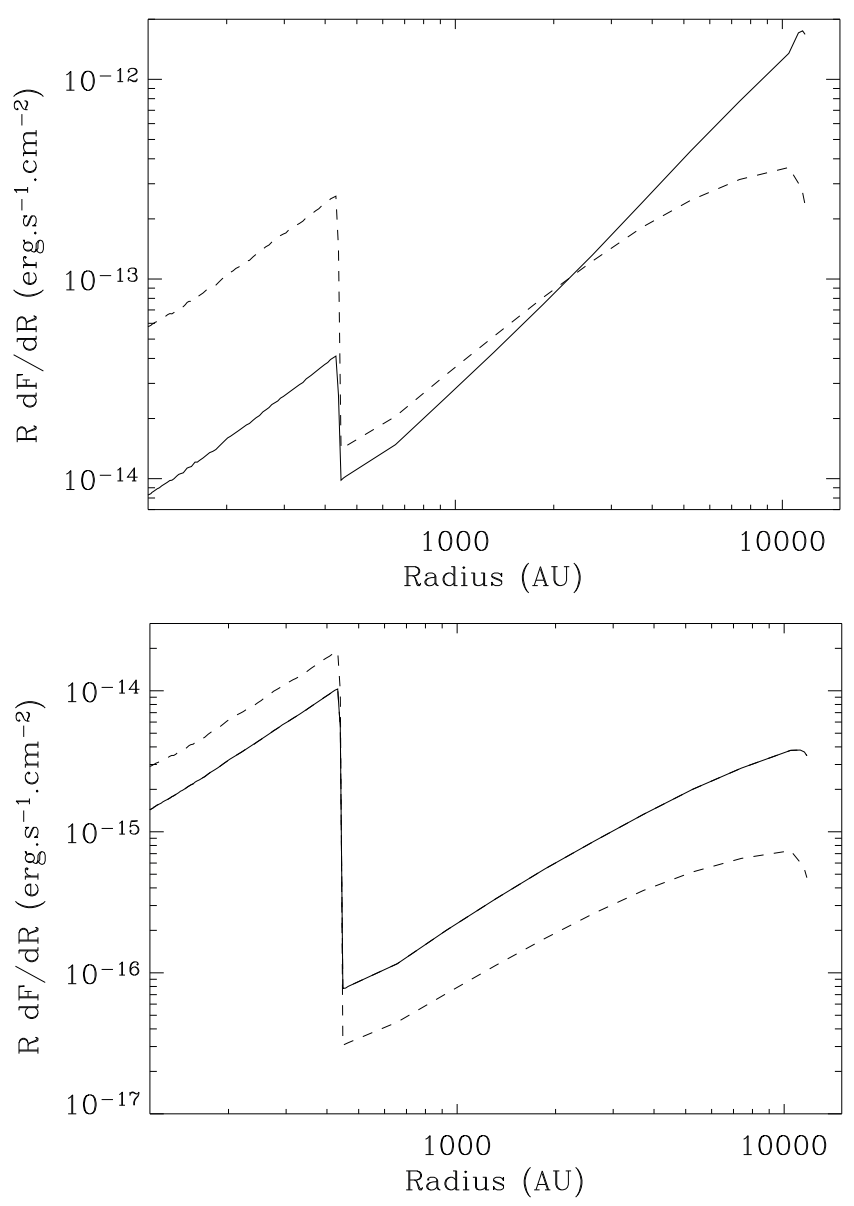

Fig. 10. Emission profile, $R \frac{\mathrm{d} F}{\mathrm{~d} R}$ of the ortho (solid line) and para (dashed line) water fundamental lines at 557 and $1113 \mathrm{GHz}$, respectively, as function of the radius, in the case of the reference model (see Table 4). The plotted quantity $R \frac{\mathrm{d} F}{\mathrm{~d} R}$ is the contribution of each shell to the flux integrated over the whole envelope. $\mathrm{H}_{2}^{16} \mathrm{O}$ and $\mathrm{H}_{2}^{18} \mathrm{O}$ emission profiles are represented in the top and bottom panels, respectively.

\subsection{Other models}

Here we explore the sensitivity of the results reported in the previous paragraph against the variation in the three main parameters of the model: the water abundance in the inner $\left(X\left(\mathrm{H}_{2} \mathrm{O}\right)_{\text {in }}\right)$ and outer $\left(X\left(\mathrm{H}_{2} \mathrm{O}\right)_{\text {out }}\right)$ envelope, and the illuminating FUV field $G_{0}$.

Figures 12 and 13 show the ratio between the line intensities of the reference model (Model 1 of Table 4) and the line intensities predicted by models with different $X\left(\mathrm{H}_{2} \mathrm{O}\right)_{\text {out }}$ and $X\left(\mathrm{H}_{2} \mathrm{O}\right)_{\text {in }}$, respectively. As noted by other authors (Ceccarelli et al. 2000; Maret et al. 2002), lines with upper level energies lower than about $200 \mathrm{~cm}^{-1}$ are sensitive to $X\left(\mathrm{H}_{2} \mathrm{O}\right)_{\text {out }}$ and insensitive to $X\left(\mathrm{H}_{2} \mathrm{O}\right)_{\text {in }}$, because these lines mostly originate in the outer envelope for excitation and line opacity reasons. A variation of a factor 10 in $X\left(\mathrm{H}_{2} \mathrm{O}\right)_{\text {out }}$ leads to an almost similar variation in the line intensity of the lowest lying lines. The higher the upper level energy, the lesser the variation. Conversely, lines with upper level energies higher than about $200 \mathrm{~cm}^{-1}$ are sensitive to $X\left(\mathrm{H}_{2} \mathrm{O}\right)_{\text {in }}$ and insensitive to $X\left(\mathrm{H}_{2} \mathrm{O}\right)_{\text {out }}$. In this case, variations by a factor 10 in $X\left(\mathrm{H}_{2} \mathrm{O}\right)_{\text {in }}$, going from $1 \times 10^{-6}$ to $1 \times 10^{-5}$, can lead to variations in the lines fluxes even 100 times greater. This extreme variation, 10 times greater than the difference in the $X\left(\mathrm{H}_{2} \mathrm{O}\right)_{\text {in }}$ variation, occurs to some lines in the $50-200 \mu \mathrm{m}$ wavelength range. This phenomenon occurs because those lines

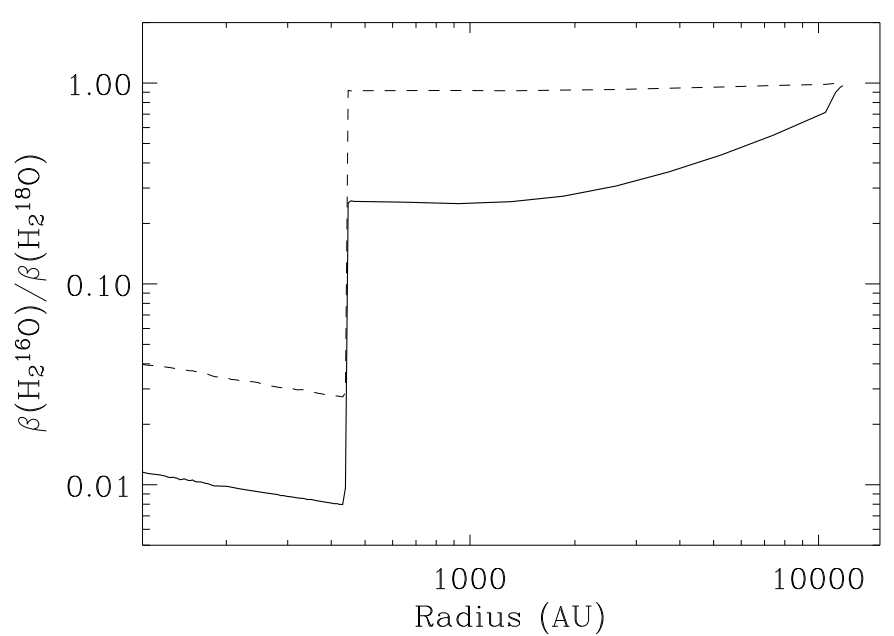

Fig. 11. Ratio of the $\mathrm{H}_{2}^{16} \mathrm{O}$ escape probability over the $\mathrm{H}_{2}^{18} \mathrm{O}$ escape probability of the ortho (solid line) and para (dashed line) water fundamental lines obtained with the reference model (model 1 of Table 4) at 557 and $1113 \mathrm{GHz}$, respectively, as function of the radius.

are in absorption rather than in emission in the region just after the ice sublimation, resulting in an additional decrease in the emerging line flux. The higher the $X\left(\mathrm{H}_{2} \mathrm{O}\right)_{\text {in }}$, the lesser the absorption depth. When $X\left(\mathrm{H}_{2} \mathrm{O}\right)_{\text {in }}$ reaches $1 \times 10^{-5}$ the absorption region generally vanishes. In addition, many high lying lines are prevalently populated by absorption of the photons emitted by the dust, so that they are particularly sensitive to the dust continuum.

Note that as mentioned in the Sect. 2.2, the inner region of the envelope is relatively unconstrained by the available observational data. Therefore we derived water line spectrum predictions by varying the power-law index of the density profile $\alpha$ of about $\sim 30 \%$ in the inner part. We observed a variation in the line intensity of a factor 5-10 for the transitions with upper level energy $\gtrsim 300-400 \mathrm{~cm}^{-1}$ and less than 2 for the lower lines. Finally, the predicted line intensities do not vary appreciably when the illuminating FUV field changes from 1 to 1000. Therefore, observations of water lines will be extremely helpful in constraining the water abundance across the envelope, but will not be sensitive to the illuminating FUV field.

\subsection{Effect of gas-dust thermal decoupling}

As presented in Sect. 3, the large quantity of water vapor injected into the gas in the inner part of the envelope causes a dramatic decoupling between the dust and gas temperatures (see Fig. 7). Obviously, this effect has a strong impact on the interpretation of the water line emission. This is illustrated in Fig. 14, where we report the ratio of the water line intensities obtained by considering the gas temperature self-consistently computed (model 1) over the case where $T_{\text {gas }}$ is assumed to be equal to $T_{\text {dust }}$. Assuming $T_{\text {gas }}=T_{\text {dust }}$ artificially leads to differences in line fluxes up to two orders of magnitude. Since the decoupling occurs in the inner part of the envelope, the higher the upper level energy, the greater the difference, for both ortho and para lines. Note that the fluxes of the two fundamental ortho and para lines are not affected by the $T_{\text {gas }}=T_{\text {dust }}$ choice. Finally, we did the same study with Model $2\left(X\left(\mathrm{H}_{2} \mathrm{O}\right)_{\text {in }}=10^{-6}\right)$. In this case, because of the smaller $X\left(\mathrm{H}_{2} \mathrm{O}\right)_{\text {in }}$, the decoupling is less significant than in the previous one, leading to changes in lines intensities up to one order of magnitude. 
Table 5. Predictions of the line fluxes (after subtraction of the continuum) of the water lines observable with the Herschel spectrometers, HIFI and PACS.

\begin{tabular}{|c|c|c|}
\hline \multicolumn{3}{|l|}{ PACS range } \\
\hline Transition & $\begin{array}{l}\text { Wavelength } \\
(\mu \mathrm{m})\end{array}$ & $\begin{array}{c}\text { Flux } \\
\left(\mathrm{erg} \mathrm{s}^{-1} \mathrm{~cm}^{-2}\right) \\
\end{array}$ \\
\hline \multicolumn{3}{|l|}{$\mathrm{H}_{2}^{16} \mathrm{O}$} \\
\hline $2_{21} \rightarrow 3_{30}$ & 66.44 & $2.99 \mathrm{E}-14$ \\
\hline $2_{20} \rightarrow 3_{31}$ & 67.09 & $1.13 \mathrm{E}-14$ \\
\hline $3_{03} \rightarrow 3_{30}$ & 67.27 & $2.28 \mathrm{E}-14$ \\
\hline $2_{12} \rightarrow 3_{21}$ & 75.38 & $1.55 \mathrm{E}-13$ \\
\hline $3_{12} \rightarrow 4_{23}$ & 78.74 & $4.51 \mathrm{E}-14$ \\
\hline $2_{11} \rightarrow 3_{22}$ & 89.99 & $5.09 \mathrm{E}-14$ \\
\hline $4_{04} \rightarrow 5_{15}$ & 95.63 & $1.05 \mathrm{E}-14$ \\
\hline $4_{14} \rightarrow 5_{05}$ & 99.49 & $4.21 \mathrm{E}-14$ \\
\hline $1_{11} \rightarrow 2_{20}$ & 100.98 & $7.80 \mathrm{E}-14$ \\
\hline $1_{10} \rightarrow 2_{21}$ & 108.07 & $1.43 \mathrm{E}-13$ \\
\hline $3_{03} \rightarrow 4_{14}$ & 113.54 & $8.45 \mathrm{E}-14$ \\
\hline $4_{23} \rightarrow 4_{32}$ & 121.72 & $1.14 \mathrm{E}-14$ \\
\hline $3_{13} \rightarrow 4_{04}$ & 125.36 & 5.34E-14 \\
\hline $4_{14} \rightarrow 4_{23}$ & 132.41 & 2.92E-14 \\
\hline $3_{21} \rightarrow 3_{30}$ & 136.49 & $1.35 \mathrm{E}-14$ \\
\hline $2_{02} \rightarrow 3_{13}$ & 138.53 & 7.84E-14 \\
\hline $3_{13} \rightarrow 3_{22}$ & 156.20 & $2.25 \mathrm{E}-14$ \\
\hline $2_{12} \rightarrow 3_{03}$ & 174.62 & 7.42E-14 \\
\hline $1_{01} \rightarrow 2_{12}$ & 179.53 & $1.17 \mathrm{E}-13$ \\
\hline $2_{12} \rightarrow 2_{21}$ & 180.49 & 4.97E-14 \\
\hline \multicolumn{3}{|l|}{ HIFI range } \\
\hline Transition & $\begin{array}{c}\text { Frequency } \\
(\mathrm{GHz})\end{array}$ & $\begin{array}{c}\begin{array}{c}\text { Flux } \\
\left(\mathrm{K} \mathrm{km} \mathrm{s}^{-1}\right)\end{array} \\
\end{array}$ \\
\hline \multicolumn{3}{|l|}{$\mathrm{H}_{2}^{16} \mathrm{O}$} \\
\hline $1_{01} \stackrel{\llcorner}{\rightarrow} 1_{10}$ & 556.96 & 14.8 \\
\hline $2_{02} \rightarrow 2_{11}$ & 752.04 & $1.06 \mathrm{E}+00$ \\
\hline $1_{11} \rightarrow 2_{02}$ & 987.95 & $1.31 \mathrm{E}+00$ \\
\hline $3_{03} \rightarrow 3_{12}$ & 1097.34 & $1.46 \mathrm{E}+00$ \\
\hline $0_{00} \rightarrow 1_{11}$ & 1113.35 & $4.32 \mathrm{E}+00$ \\
\hline $2_{21} \rightarrow 3_{12}$ & 1153.09 & $2.50 \mathrm{E}+00$ \\
\hline $3_{12} \rightarrow 3_{21}$ & 1162.93 & $6.97 \mathrm{E}-01$ \\
\hline $2_{11} \rightarrow 2_{20}$ & 1228.81 & $5.33 \mathrm{E}-01$ \\
\hline $4_{13} \rightarrow 4_{22}$ & 1207.62 & $2.45 \mathrm{E}-01$ \\
\hline $5_{14} \rightarrow 5_{23}$ & 1410.65 & $1.47 \mathrm{E}-01$ \\
\hline $2_{12} \rightarrow 2_{21}$ & 1660.99 & $2.33 \mathrm{E}+00$ \\
\hline $4_{04} \rightarrow 4_{13}$ & 1602.23 & $1.46 \mathrm{E}-01$ \\
\hline $1_{01} \rightarrow 2_{12}$ & 1669.87 & $5.46 \mathrm{E}+00$ \\
\hline $2_{12} \rightarrow 3_{03}$ & 1716.83 & $3.37 \mathrm{E}+00$ \\
\hline $\begin{array}{c}5_{23} \rightarrow 5_{32} \\
\mathrm{H}_{2}^{18} \mathrm{O}\end{array}$ & 1867.75 & 3.13E-02 \\
\hline $1_{01} \rightarrow 1_{10}$ & 556.96 & $1.88 \mathrm{E}-01$ \\
\hline $0_{00} \rightarrow 1_{11}$ & 1113.35 & $1.48 \mathrm{E}-01$ \\
\hline
\end{tabular}

The predictions refer to the reference model (model 1 of Table 4).

\subsection{Constraints from ISO data}

Observations of FIR4 were obtained by the spectrometer ISOLWS in the grating mode (spectral resolution 200) and FabryPerot (spectral resolution $\sim 10^{4}$ ). We retrieved the data from the ISO Data Archive (http://iso.esac.esa.int/ida/). No water lines were detected. Table 6 summarizes the upper limits obtained for the predicted brightest lines, together with the predictions of the reference model and model 6. Unfortunately, the ISO sensitivity is not enough to put noticeables constraints on the water abundance across the FIR4 envelope.
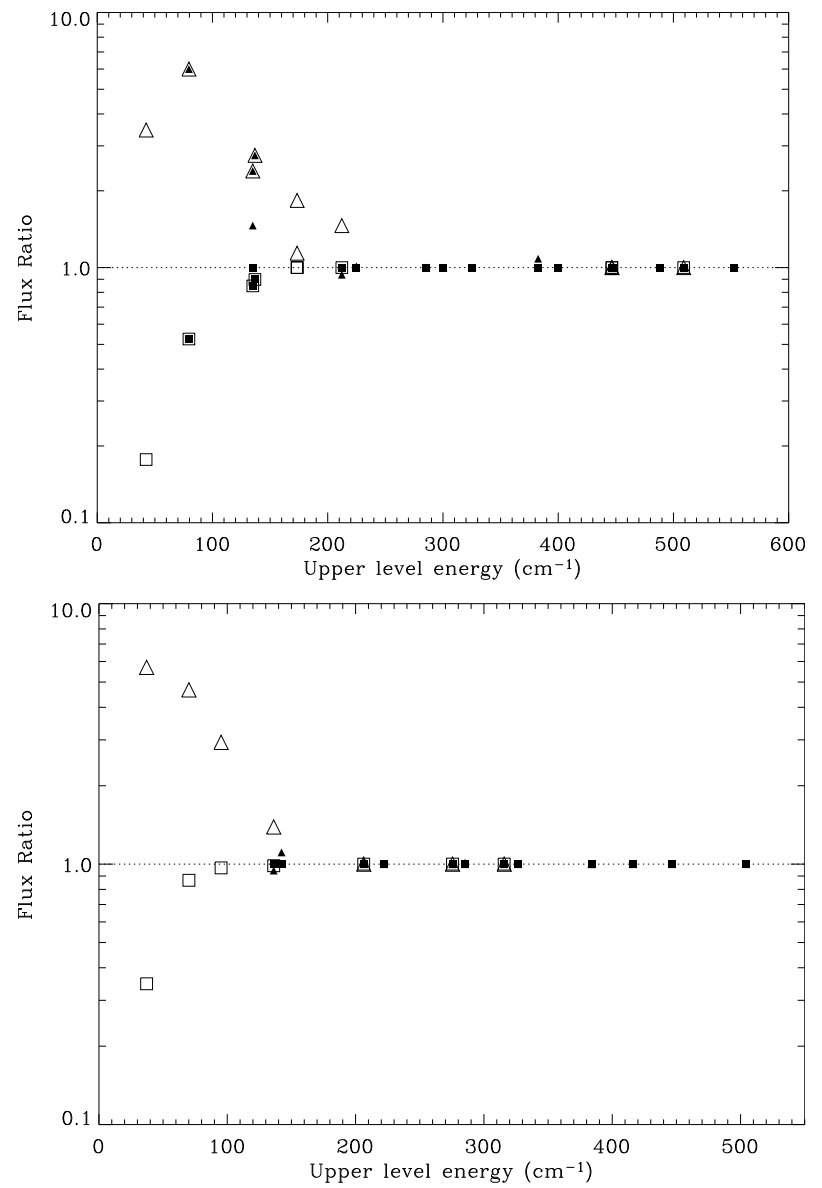

Fig. 12. Ratio between the line fluxes of the reference model (model 1 of Table 4) and the line fluxes predicted by models with same $X\left(\mathrm{H}_{2} \mathrm{O}\right)_{\text {in }}$ but different $X\left(\mathrm{H}_{2} \mathrm{O}\right)_{\text {out }}$. Flux ratios between model 4 (model 5) and model 1 are represented by squares (triangles). Filled and empty symbols refer to the lines emitted in the PACS and HIFI bands, respectively. The upper (lower) panel reports ortho (para) water line intensities ratios.

\section{Concluding remarks}

We analyzed in great detail the continuum emission from the IM protostar OMC2-FIR4, with the aim of deriving the physical structure of its envelope, a mandatory first step for further studies to understand the formation process. Our analysis led to a new estimate of the FIR4 luminosity, which is $1000 L_{\odot}$. The density of the envelope surrounding FIR4 has a shallow dependence on the radius, the density power-law index being only 0.6 . Since systematic studies of the IM protostars envelopes have not been published yet, we can tentatively compare the FIR4 envelope with low- and high-mass protostellar envelopes, where similar studies have been carried out. Specifically, Jørgensen et al. (2002) analyzed 18 Class 0 and I sources and found that the average power-law index $\alpha$ in Class 0 sources is $1.3 \pm 0.4$, while in Class I sources it is $1.7 \pm 0.1$, significatively greater than the value we found in FIR4. Similarly, van der Tak et al. (2000) studied a sample of high-mass protostars and found $\alpha=$ $1.4 \pm 0.4$. One has to notice that there are exceptions, however, with sources in both low and high-mass showing a smaller than unity $\alpha$ value: L1527 $(\alpha=0.6)$ and L483 $(\alpha=0.9)$ in the Class 0 sources, GL7009S $(\alpha=0.5)$ in the high-mass protostars sample. It is not clear what makes these sources "anomalous": the presence of strong asymmetries (Jørgensen et al. 2002) have been suggested as a possible reason. The case of FIR4 seems to fall in 
Table 6. The brightest lines predicted by models 1 and 6 (the model with the largest water abundance) compared with the upper limits derived by the ISO observations.)

\begin{tabular}{cccccc}
\hline \hline $\begin{array}{c}\text { Wavelength } \\
(\mu \mathrm{m})\end{array}$ & $\begin{array}{c}\text { Transition } \\
J_{K_{-} K_{+}} \rightarrow J_{K_{-}^{\prime} K_{+}^{\prime}}^{\prime}\end{array}$ & $\begin{array}{c}\text { Model 1 } \\
\text { Intensity } \\
\left(\mathrm{erg} \mathrm{s}^{-1} \mathrm{~cm}^{-2}\right)\end{array}$ & $\begin{array}{c}\text { Model 6 } \\
\text { Intensity } \\
\left(\mathrm{erg} \mathrm{s}^{-1} \mathrm{~cm}^{-2}\right)\end{array}$ & $\begin{array}{c}\text { ISO } \\
\text { Instrument }\end{array}$ & $\begin{array}{c}\text { Upper limit }(3 \sigma) \\
\left(\mathrm{erg} \mathrm{s}^{-1} \mathrm{~cm}^{-2}\right)\end{array}$ \\
\hline $\begin{array}{c}\text { ortho } \\
75.4957\end{array}$ & $8_{54} \rightarrow 8_{45}$ & $2.6 \mathrm{E}-20$ & $1.9 \mathrm{E}-19$ & LWS04 & $9.78 \mathrm{e}-12$ \\
108.073 & $2_{21} \rightarrow 1_{10}$ & $1.3 \mathrm{E}-12$ & $1.5 \mathrm{E}-12$ & LWS04 & $2.21 \mathrm{e}-12$ \\
179.527 & $2_{12} \rightarrow 1_{01}$ & $1.2 \mathrm{E}-12$ & $7.0 \mathrm{E}-12$ & LWS04 & $2.56 \mathrm{e}-11$ \\
113.538 & $4_{14} \rightarrow 3_{03}$ & $8.3 \mathrm{E}-13$ & $8.7 \mathrm{E}-13$ & LWS01 & $1.2 \mathrm{e}-11$ \\
para & & & & & \\
100.983 & $2_{20} \rightarrow 1_{11}$ & $7.3 \mathrm{E}-13$ & $9.1 \mathrm{E}-13$ & LWS01 & $8.37 \mathrm{e}-12$ \\
138.527 & $3_{13} \rightarrow 2_{02}$ & $7.3 \mathrm{E}-13$ & $7.3 \mathrm{E}-13$ & LWS01 & $4.14 \mathrm{e}-12$ \\
156.197 & $3_{22} \rightarrow 3_{13}$ & $2.2 \mathrm{E}-13$ & $3.4 \mathrm{E}-13$ & LWS01 & $1.60 \mathrm{e}-12$ \\
\hline
\end{tabular}
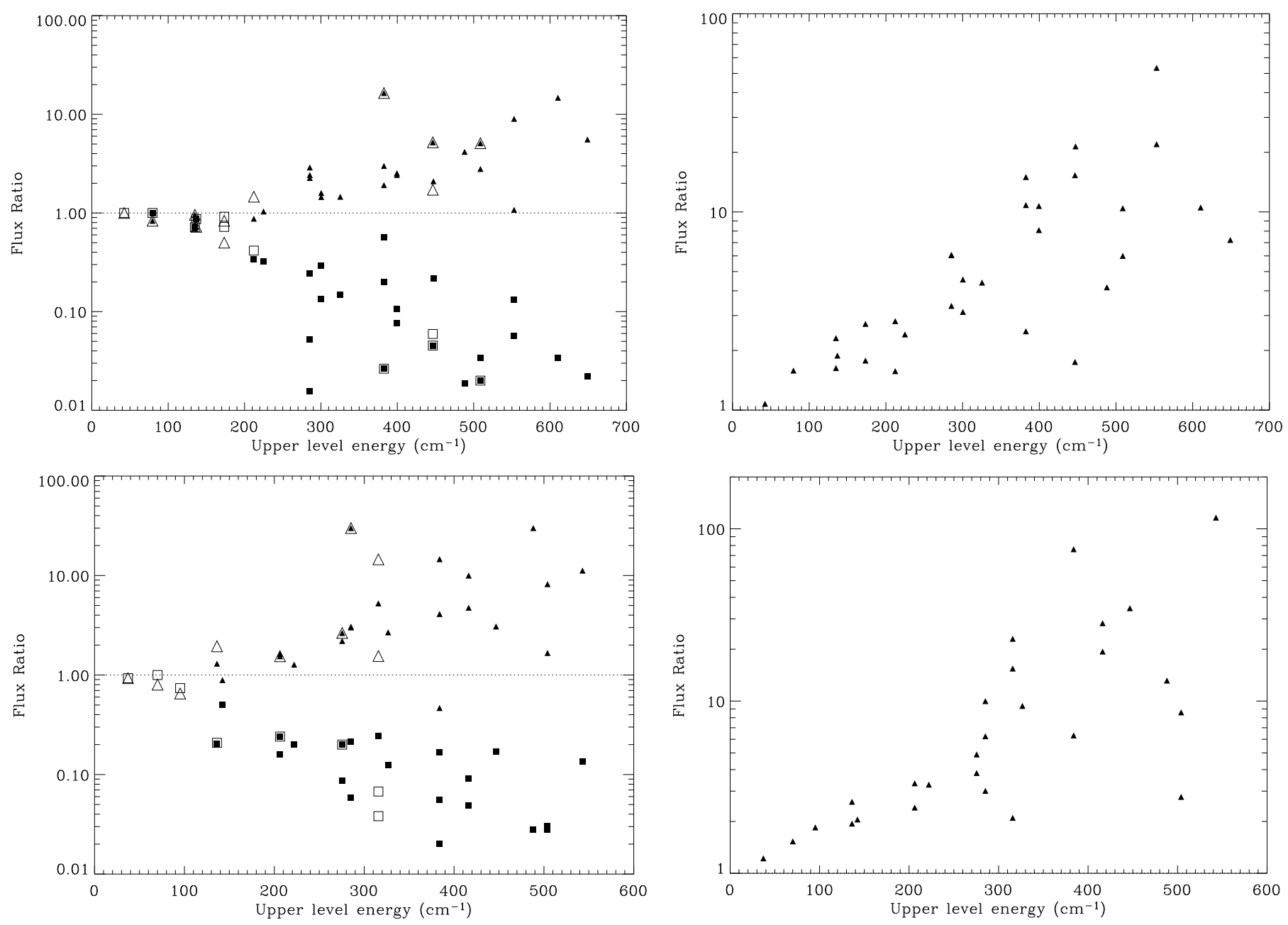

Fig. 13. Ratio between the line fluxes of the reference model (model 1 of Table 4) and the line fluxes predicted by models with same $X\left(\mathrm{H}_{2} \mathrm{O}\right)_{\text {out }}$ but different $X\left(\mathrm{H}_{2} \mathrm{O}\right)_{\text {in }}$. Flux ratios between model 2 (model 3) and model 1 are represented by squares (triangles). Filled and empty symbols refer to the lines emitted in the PACS and HIFI bands, respectively. The upper (lower) panel reports ortho (para) water line flux ratios.

this "anomalous sources" category, and further studies, possibly on chemistry, are needed to say more.

Given the suggestion by Jørgensen et al. (2006) that a strong FUV field $\left(G_{0}=1 \times 10^{4}\right)$ illuminates the FIR4 envelope, we explored the cases of different FUV fields. As already noted by the

Fig. 14. Ratios between the line fluxes of the reference model (model 1) and the reference model with gas-dust non-thermally decoupled (namely $T_{\text {gas }}=T_{\text {dust }}$, as a function of the upper level energy of the transition. Upper and lower panels show ortho and para $\mathrm{H}_{2} \mathrm{O}$ lines, respectively.

same authors, however, the dust continuum cannot really distinguish whether a strong illuminating FUV field is impinging on the envelope. In fact, Jørgensen et al. (2006) adopted a steeper density distribution $(\alpha=2)$ that allows the FUV photons to penetrate deeper into the envelope. Their conclusions were based on submillimeter lines from $\mathrm{CO}$ and $\mathrm{H}_{2} \mathrm{CO}$, which would be 
exceedingly bright if they were emitted in the envelope. They attributed the lines to the warm gas at the border of the envelope, heated up by the hypothetical large FUV field. However, as discussed in Sect. 2.3, OI and CII maps by Herrmann et al. (1997) show that the entire OMC2 region is illuminated by a $G_{0}=500$ FUV field, which would imply an even lower FUV field on the FIR4 envelope. One has also to notice here that large scale maps by Schloerb \& Loren (1982) show that the CO $(1 \rightarrow 0)$ line is bright $(\sim 40 \mathrm{~K})$ over the whole OMC2 region, a fact that led Herrmann et al. (1997) to attribute the CO emission to the PDR associated with the cloud. In addition, several outflows are known to "pollute" the $\mathrm{CO}$ emission in the region, in particular the one originating from FIR3 (25" North of FIR4: Fig. 1) and reaching FIR4 and FIR5 (Williams et al. 2003). All the above considerations together lead to the conclusion that the FUV field impinging FIR4 is not anomalously large and less than 500. Therefore, given the presence of a bright PDR and a "polluting" outflow from FIR3, caution is needed in interpreting the low lying water lines, as much as lines from any molecule, separately from the whole molecular cloud emission.

One major motivation of the present work is to predict of the water line spectrum from FIR4, as this source will be observed in the 500-2000 GHz frequency range by the incoming Herschel Space Observatory (FIR4 is a target of the Key Program "HIFI Spectral Surveys of Star Formation Regions": http: //www-laog.obs.ujf-grenoble.fr/heberges/ hs $3 \mathrm{f} /$ ). In the present study, we have shown that water is indeed a key molecular species, because of its strong impact on the gas cooling in the region where the dust temperature exceeds $100 \mathrm{~K}$, the sublimation temperature of the dust grain ices. The large quantity of water vapor injected into the gas by the sublimated ices very efficiently cools the gas, causing a dramatic decoupling between the dust and gas temperatures. Depending on the abundance of the injected water vapor, the difference in the temperature can be as high as $50 \mathrm{~K}$ at the sublimation radius (namely 50\%!) and even higher going inward. For example, at $100 \mathrm{AU}$ the dust temperature is predicted to be around $280 \mathrm{~K}$, whereas the gas temperature is $80 \mathrm{~K}$ if the water abundance is $1 \times 10^{-4}$. Obviously, this has a strong impact in the interpretation of the water line emission, as much as, the emission from any molecular species emitting in the inner region. In fact, the comparison of the water line emission between the case where dust and gas are assumed to be thermally coupled and the case where the gas temperature is self-consistently computed shows that the difference in the line intensity can reach two orders of magnitudes for lines with large upper level energies (namely the lines excited in the innermost region, where gas and dust decouple). Therefore, our important second conclusion is that caution has to be applied in interpreting the line emission from FIR4, as much as any source with a similar luminosity and envelope structure. Gas and dust temperature can be very different, and to derive correct molecular abundances (including water abundance), they have to be both estimated, accounting for all terms of heating and cooling. Avoiding that may lead to very wrong conclusions.

Acknowledgements. We warmly thank Moshe Elitzur for his valuable help in using the DUSTY code. We also wish to thank Neal Evans and Doug Johnstone for helpful discussions, and Doug Johnstone and Darek Lis for providing us with the JCMT and CSO continuum maps of the OMC2-FIR4 region. We thank an anonymous referee and Malcolm Walmsley for comments that helped in improving the manuscript. One of us (N.Crimier) is supported by a fellowship of the Ministère de l'Enseignement Supérieur et de la Recherche.

\section{References}

Ali, B., \& Depoy, D. L. 1995, AJ, 109, 709

Aso, Y., Tatematsu, K., Sekimoto, Y., et al. 2000, ApJS, 131, 465

Beuther, H., Churchwell, E. B., McKee, C. F., \& Tan, J. C. 2007, in Protostars and Planets V, ed. B. Reipurth, D. Jewitt, \& K. Keil, 165

Black, J. H. 1994, in The First Symposium on the Infrared Cirrus and Diffuse Interstellar Clouds. Astron. Soc. Pac., San Francisco, ed. R. M. Cutri, W. B. Latter, ASP Conf. Ser., 58, 355

Ceccarelli, C., Hollenbach, D. J., \& Tielens, A. G. G. M. 1996, ApJ, 471, 400

Ceccarelli, C., Castets, A., Caux, E., et al. 2000, A\&A, 355, 1129

Ceccarelli, C., Maret, S., Tielens, A. G. G. M., Castets, A., \& Caux, E. 2003, A\&A, 410, 587

Ceccarelli, C., Caselli, P., Herbst, E., Tielens, A. G. G. M., \& Caux, E. 2007, in Protostars and Planets V, ed. B. Reipurth, D. Jewitt, \& K. Keil, 47

Chini, R., Reipurth, B., Ward-Thompson, D., et al. 1997, ApJ, 474, L135

Di Francesco, J., Evans, II, N. J., Harvey, P. M., et al. 1997, ApJ, 482, 433

Di Francesco, J., Evans, II, N. J., Caselli, P., et al. 2007, in Protostars and Planets

V, ed. B. Reipurth, D. Jewitt, \& K. Keil, 17

Doty, S. D., \& Neufeld, D. A. 1997, ApJ, 489, 122

Draine, B. T. 1978, ApJS, 36, 595

Dubernet, M.-L., Daniel, F., Grosjean, A., \& Lin, C. Y. 2009, A\&A, 497, 911

Evans, II, N. J., Rawlings, J. M. C., Shirley, Y. L., \& Mundy, L. G. 2001, ApJ, 557,193

Faure, A., \& Josselin, E. 2008, A\&A, 492, 257

Faure, A., Crimier, N., Ceccarelli, C., et al. 2007, A\&A, 472, 1029

Fuente, A., Ceccarelli, C., Neri, R., et al. 2007, A\&A, 468, L37

Gatley, I., Becklin, E. E., Mattews, K., et al. 1974, ApJ, 191, L121+

Gomez, M., Hartmann, L., Kenyon, S. J., \& Hewett, R. 1993, AJ, 105, 1927

Gondhalekar, P. M., \& Wilson, R. 1975, A\&A, 38, 329

Habing, H. J. 1968, Bull. Astron. Inst. Netherlands, 19, 421

Herrmann, F., Madden, S. C., Nikola, T., et al. 1997, ApJ, 481, 343

Hillenbrand, L. A., \& Hartmann, L. W. 1998, ApJ, 492, 540

Hirota, T., Bushimata, T., Choi, Y. K., et al. 2007, PASJ, 59, 897

Hunter, T. R., Benford, D. J., \& Serabyn, E. 1996, PASP, 108, 1042

Ivezic, Z., \& Elitzur, M. 1997, MNRAS, 287, 799

Johnson, J. J., Gehrz, R. D., Jones, T. J., Hackwell, J. A., \& Grasdalen, G. L. 1990, AJ, 100, 518

Johnstone, D., \& Bally, J. 1999, ApJ, 510, L49

Johnstone, D., Boonman, A. M. S., \& van Dishoeck, E. F. 2003, A\&A, 412, 157

Jørgensen, J. K., Schöier, F. L., \& van Dishoeck, E. F. 2002, A\&A, 389, 908

Jørgensen, J. K., Johnstone, D., van Dishoeck, E. F., \& Doty, S. D. 2006, A\&A, 449,609

Lis, D. C., Serabyn, E., Keene, J., et al. 1998, ApJ, 509, 299

Mannings, V., \& Sargent, A. I. 1997, ApJ, 490, 792

Mannings, V., \& Sargent, A. I. 2000, ApJ, 529, 391

Maret, S., Ceccarelli, C., Caux, E., Tielens, A. G. G. M., \& Castets, A. 2002, A\&A, 395, 573

Mezger, P. G., Zylka, R., \& Wink, J. E. 1990, A\&A, 228, 95

Neri, R., Fuente, A., Ceccarelli, C., et al. 2007, A\&A, 468, L33

Öberg, K. I., van Broekhuizen, F., Fraser, H. J., et al. 2005, ApJ, 621, L33

Ossenkopf, V., \& Henning, T. 1994, A\&A, 291, 943

Parise, B., Ceccarelli, C., \& Maret, S. 2005, A\&A, 441, 171

Reipurth, B., Rodríguez, L. F., \& Chini, R. 1999, AJ, 118, 983

Sandell, G., \& Weintraub, D. A. 2001, ApJS, 134, 115

Schloerb, F. P., \& Loren, R. B. 1982, New York Academy Sciences Annals, 395, 32

Schöier, F. L., Jørgensen, J. K., van Dishoeck, E. F., \& Blake, G. A. 2002, A\&A, 390, 1001

Shirley, Y. L., Evans, II, N. J., \& Rawlings, J. M. C. 2002, ApJ, 575, 337

Takahashi, T., Silk, J., \& Hollenbach, D. J. 1983, ApJ, 275, 145

Testi, L., \& Sargent, A. I. 1998, ApJ, 508, L91

Testi, L., Palla, F., \& Natta, A. 1999, A\&A, 342, 515

van der Tak, F. F. S., van Dishoeck, E. F., Evans, II, N. J., Bakker, E. J., \& Blake, G. A. 1999, ApJ, 522, 991

van der Tak, F. F. S., van Dishoeck, E. F., Evans, II, N. J., \& Blake, G. A. 2000, ApJ, 537, 283

Williams, J. P., Plambeck, R. L., \& Heyer, M. H. 2003, ApJ, 591, 1025

Wu, Y., Zhang, Q., Chen, H., et al. 2005, AJ, 129, 330

Young, C. H., \& Evans, II, N. J. 2005, ApJ, 627, 293

Young, C. H., Shirley, Y. L., Evans, II, N. J., \& Rawlings, J. M. C. 2003, ApJS, 145,111 